OPEN ACCESS

Edited by: Mukesh Prasad, University of Technology Sydney, Australia

Reviewed by:

Sharon Qi,

UCLA, United States

Diane Schott,

University of Nebraska Medical

Center, United States

${ }^{*}$ Correspondence: Jake Kendrick

jake.kendrick@research.uwa.edu.au

Specialty section: This article was submitted to Cancer Imaging and Image-directed Interventions, a section of the journal Frontiers in Oncology

Received: 07 September 2021 Accepted: 11 October 2021 Published: 01 November 2021

Citation:

Kendrick J, Francis R, Hassan GM, Rowshanfarzad P, Jeraj R, Kasisi $C$ Rusanov B and Ebert M (2021) Radiomics for Identification and Prediction in Metastatic Prostate Cancer: A Review of Studies. Front. Oncol. 11:771787. doi: 10.3389/fonc.2021.771787

\section{Radiomics for Identification and Prediction in Metastatic Prostate Cancer: A Review of Studies}

\author{
Jake Kendrick $^{1 *}$, Roslyn Francis ${ }^{2,3}$, Ghulam Mubashar Hassan ${ }^{1}$, Pejman Rowshanfarzad ${ }^{1}$, \\ Robert Jeraj ${ }^{4,5}$, Collin Kasisi ${ }^{3}$, Branimir Rusanov ${ }^{1}$ and Martin Ebert ${ }^{1,6,7}$ \\ 1 School of Physics, Mathematics and Computing, University of Western Australia, Perth, WA, Australia, 2 Medical School, \\ University of Western Australia, Crawley, WA, Australia, ${ }^{3}$ Department of Nuclear Medicine, Sir Charles Gairdner Hospital, \\ Perth, WA, Australia, ${ }^{4}$ Department of Medical Physics, University of Wisconsin, Madison, WI, United States, ${ }^{5}$ Faculty of \\ Mathematics and Physics, University of Ljubljana, Ljubljana, Slovenia, ${ }^{6}$ Department of Radiation Oncology, Sir Charles \\ Gairdner Hospital, Perth, WA, Australia, 750 Clinics, Claremont, WA, Australia
}

Metastatic Prostate Cancer (mPCa) is associated with a poor patient prognosis. $\mathrm{mPCa}$ spreads throughout the body, often to bones, with spatial and temporal variations that make the clinical management of the disease difficult. The evolution of the disease leads to spatial heterogeneity that is extremely difficult to characterise with solid biopsies. Imaging provides the opportunity to quantify disease spread. Advanced image analytics methods, including radiomics, offer the opportunity to characterise heterogeneity beyond what can be achieved with simple assessment. Radiomics analysis has the potential to yield useful quantitative imaging biomarkers that can improve the early detection of $\mathrm{mPCa}$, predict disease progression, assess response, and potentially inform the choice of treatment procedures. Traditional radiomics analysis involves modelling with hand-crafted features designed using significant domain knowledge. On the other hand, artificial intelligence techniques such as deep learning can facilitate end-to-end automated feature extraction and model generation with minimal human intervention. Radiomics models have the potential to become vital pieces in the oncology workflow, however, the current limitations of the field, such as limited reproducibility, are impeding their translation into clinical practice. This review provides an overview of the radiomics methodology, detailing critical aspects affecting the reproducibility of features, and providing examples of how artificial intelligence techniques can be incorporated into the workflow. The current landscape of publications utilising radiomics methods in the assessment and treatment of mPCa are surveyed and reviewed. Associated studies have incorporated information from multiple imaging modalities, including bone scintigraphy, CT, PET with varying tracers, multiparametric MRI together with clinical covariates, spanning the prediction of progression through to overall survival in varying cohorts. The methodological quality of 
each study is quantified using the radiomics quality score. Multiple deficits were identified, with the lack of prospective design and external validation highlighted as major impediments to clinical translation. These results inform some recommendations for future directions of the field.

Keywords: radiomics, metastatic prostate cancer, radiomics quality score, deep learning, PET, CT, MRI

\section{INTRODUCTION}

Prostate Cancer (PCa) is a pernicious disease that is one of the leading causes of cancer death among men throughout the world (1). Early-stage diagnosis yields high 5-year survival rates of above $90 \%$, however, once the disease metastasises, it becomes very lethal as 5-year survival rates drop drastically to less than $40 \%$ (2). For patients with bone metastases (BM), which is one of the most common sites of metastases in PCa (3), 5-year survival rates drop even further to less than $10 \%$ (4). Lymph node involvement (LNI) also yields a poor prognosis for patients (5). The clinical management of the disease is complicated by its heterogeneity (6), with current practice aiming to stratify patients into risk categories such that high-risk lesions are identified and treated, and the over-treatment of low-risk lesions is minimized (7). Radical prostatectomy (RP) is a prominent treatment for localised disease, however, between 20 and $40 \%$ of patients presents with biochemical recurrence (BCR) with the possibility of developing subsequent metastasis (8). The early identification of localised PCa patients at high risk of developing subsequent nodal or distant metastases is thus crucially important and can substantially affect the clinical decision-making process to the potential benefit of the patient (9). Established techniques for PCa diagnosis and risk stratification such as the digital rectal examination (DRE), prostate-specific antigen (PSA) test, and transrectal ultrasound (TRUS)-guided biopsy have significant limitations. DRE suffers from a high false positive rate (10), while PSA is a non-specific blood biomarker that can be elevated even in the absence of PCa (11), and even low levels do not preclude the presence of high- or medium-grade PCa (12). TRUS-guided biopsy is typically conducted via random sampling and fails to capture the heterogeneity inherent in the lesion. Furthermore, analysis of post RP specimens has demonstrated that the Gleason Score (GS) obtained from the pre-treatment needle biopsy often differs from that obtained on the final RP specimen (13).

The potential for non-invasive assessment of PCa risk, metastatic potential, and even treatment response, has been facilitated by the advance of medical imaging technologies in recent decades. Medical imaging modalities such as multiparametric magnetic resonance imaging (mpMRI), positron emission tomography (PET) and computed tomography (CT) play an important role in the diagnosis and management of localised and metastatic prostate cancer ( $\mathrm{mPCa}$ ). Bone scintigraphy is an important imaging modality that is commonly used to diagnose the extent of bone metastasis in mPCa patients (14). mpMRI is the most important imaging modality in the initial detection and staging of localised PCa due to its superior soft tissue contrast and high resolution and can localise areas of suspicion for subsequent biopsy $(15,16)$. PET radiotracers that target prostate specific membrane antigen (PSMA) such as ${ }^{68} \mathrm{Ga}$-PSMA are quickly becoming the standard of care when it comes to the management of biochemically recurrent $\mathrm{PCa}$ following definitive primary therapy. PSMA tracers enable detection of suspicious lesions because they target directly the PSMA receptor which is vastly overexpressed in the majority of PCa cases (17-19). The quantitative, rather than qualitative, analysis of each of these imaging modalities to identify unique diagnostic or prognostic biomarkers has the potential to become the basis for a personalised medicine approach to patient treatment.

Radiomics is defined as the extraction of large numbers of imaging features from medical images to quantify specific tumour attributes and phenotypes, with the ultimate goal of utilising these features to glean valuable diagnostic or prognostic information that can inform clinical decision making (20). The central hypothesis of radiomics is that these extracted mathematical features are reflective of the underlying tumour biology, and that they can therefore be used to guide treatment procedures and advance personalised therapy on a patient-topatient basis (21). Typically, images are visually assessed by radiologists whose qualitative observations are highly variable (22-24). The extraction of radiomics features, combined with a sufficiently quantitative image acquisition, enables a more quantitative and objective characterisation of the tumour which can overcome this inter-observer variability, and potentially yield useful predictive biomarkers that cannot be discerned via visual analysis. The radiomics approach has the advantage of being non-invasive, as opposed to other techniques such as biopsy which, in addition to being invasive (25), are limited in their capacity to characterise the spatial and temporal heterogeneity of lesions (26). Non-invasive assessment of intratumoral heterogeneity is highly desirable since it is a known factor affecting disease progression and response (27). Furthermore, medical imaging scans are a part of the conventional clinical management scheme for most patients, meaning that radiomics models can typically be incorporated clinically without adding any significant burden to the existing workflow (28).

Despite the enormous potential of radiomics to facilitate individualised patient therapy, the process does come with associated challenges. The radiomics workflow contains a myriad of factors that can profoundly affect the resulting quantitative imaging biomarker measurement; anything from the algorithm used to reconstruct the medical image to the interpolation method utilised in an up- or down-sampling 
procedure can introduce variability into radiomics research (29, 30). The sensitivity of extracted features to a host of procedural factors has contributed to poor scientific reproducibility in radiomics research that substantially affects its translational capacity (31). Recognising the need to ensure the scientific rigour of radiomics studies, Lambin et al. (32) introduced the Radiomics Quality Score (RQS), a points-based system that rewards and penalises radiomics papers according to specific attributes of their methodologies. Modelled on the Transparent Reporting of a multi-variable prediction model for Individual Prognosis or Diagnosis (TRIPOD) initiative (33), the RQS identifies key aspects of the radiomics workflow, such as the necessity of reporting imaging parameters in a comprehensive fashion, conducting external validation of the results and the nature of the study (retrospective/prospective), and generates a score out of 36 providing an indication of how scientifically rigorous the study is. The RQS, despite some limitations, is a useful way to identify methodological weaknesses in reported radiomics studies. The quality of radiomics reporting, as measured by the RQS, in oncological studies in general is poor (34), and a plethora of studies have demonstrated that this fact generalises across a range of malignancies and modalities such as liver metastases (35), prostate cancer MRI studies (36), neurooncologic studies (37), and non-small cell lung cancer radiomics research (38). No such analysis has been undertaken for radiomics studies pertaining to $\mathrm{mPCa}$.

This review aims to, (i) provide a methodological overview of the radiomics workflow with comments on how artificial intelligence (AI) techniques can complement the process, (ii) elucidate the current landscape of literature pertaining to how radiomics models can potentially be utilised in the clinical management of $\mathrm{mPCa}$ while providing a quality assessment in the form of a RQS and, (iii) comment on the limitations of the field and offer recommendations on future research.

\section{RADIOMICS: A METHODOLOGICAL OVERVIEW}

The typical radiomics workflow consists of several defined steps, including: (i) Medical image acquisition and reconstruction; (ii) Region of Interest (ROI) segmentation; (iii) post-processing of the acquired image; (iv) feature extraction; (v) feature selection; and (vi) model development. These steps are summarised in Figure 1. Critical factors affecting the numerical output of each feature will be discussed where appropriate as each step is outlined sequentially below. The applicability of AI methods and how they can substantively aid the process will also be discussed.

\subsection{Image Acquisition/Reconstruction}

The first step in the radiomics pipeline is the acquisition of a medical image, which becomes the basis for the analysis conducted throughout the rest of the process. The acquisition and reconstruction of medical images is subject to significant variability both within and between different institutions. In theory, any parameter that will affect the output distribution of voxel intensities will affect the calculated feature values, and thus the medical image acquisition and reconstruction parameters will greatly affect the outcome of the feature extraction process. This remains true regardless of the medical imaging modality used on the patient and can have a significant effect on the reproducibility of radiomics studies: features that demonstrate clinical relevance in one clinical setting may not be useful in a separate institution where a different imaging protocol is used. The slice thickness and pixel spacing of reconstructed medical images, for example, are known factors affecting radiomics feature output values $(39,40)$. A recent systematic review identified the reconstruction algorithm, number of iterations, and the level of gaussian smoothing as factors also affecting biomarker reproducibility for PET scans (41). When acquiring CT scans of patients, acquisition variables such as the tube current and voltage $(42,43)$, the pitch $(44)$, and even the vendor of the scanner can affect the numerical output of calculated features $(45,46)$. MRI is particularly challenging as a modality since voxel intensity values are not standardised and can vary greatly depending on the acquisition parameters chosen (47), and studies have indeed demonstrated that some of these parameters, such as image noise (48), choice of reconstruction algorithm (49), dynamic range and matrix size (50), do affect the output feature values. Figure 1 displays a non-exhaustive list of the critical factors to consider that will affect biomarker outputs at each step in the radiomics workflow. The large dependence of radiomics features on acquisition and reconstruction protocols makes it imperative that these protocols are extensively documented when presenting the results of radiomics research to maximise study reproducibility (32).

\subsection{ROI Segmentation}

A precise delineation of the ROI is a requirement for input into the feature extraction algorithm. The image voxels within this ROI define the anatomical/physiological area from which the features will be extracted in subsequent steps in the radiomics workflow; therefore, any variability in this segmentation will affect the numerical output for each feature. Segmentation of ROI's can be done manually, semi-automatically or automatically. Manual segmentations performed by clinical experts are typically used, however, performing this task manually has well documented limitations such as interobserver variability and a significant clinical time burden, which limits its feasibility for radiomics analyses in larger datasets $(51,52)$. Variability in manual segmentations has the effect of introducing bias into the evaluation of quantitative imaging biomarkers (53). Efforts should be taken to mitigate against this bias by performing multiple segmentations. Feature robustness analyses should be conducted both between multiple independent manual observer segmentations, and also between manual segmentations and semi- or fully automated algorithms, where possible (32).

\subsection{Image Post-Processing}

Prior to radiomics feature analysis, there are a number of imaging post-processing steps that are typically conducted. Image discretization is one of these steps, involving the 




FIGURE 1 | Overview of the radiomics workflow, with a non-exhaustive list of some critical factors affecting feature reproducibility.

discretization of image voxel intensities from a continuous spectrum into a set of discrete intensity bins. Limiting the range of intensity values is necessary to make the calculation of subsequent radiomics features computationally tractable (22). It also has the benefit of noise reduction (54). There are two discretisation schemes available to radiomics researchers, namely, fixed bin number and fixed bin width, and the method chosen can affect the quantitative metrics subsequently extracted. A plethora of studies have demonstrated that the intensity discretisation scheme used can affect the reproducibility of radiomics features, and thus potentially affect the predictive model derived from them (40,55-59). This fact, among many others discussed below, underscores the critical importance of transparent and comprehensive reporting of post-processing steps undertaken in radiomics studies.

Resampling image voxels to isotropic spacing is another necessary post-processing technique. Isotropic voxel spacing is necessary to ensure that the extracted texture features are rotationally invariant (54). Moreover, in-plane and throughplane spatial resolutions of medical images are commonly not unified across patient scans in radiomics datasets which can affect output feature values; therefore, resampling to a common spatial voxel resolution can be employed in an attempt to increase the reproducibility of feature values (30). There is as yet no consensus on whether up-sampling or down-sampling is preferred. Down-sampling images to a lower spatial resolution will necessarily result in information loss, whereas up-sampling images will result in the addition of false information. Different interpolation techniques exist to resample images to isotropic voxel spacing, such as nearest neighbour, trilinear, and cubic BSpline; the method chosen can have a significant impact on the reproducibility of radiomic features. Recent studies have demonstrated that the chosen interpolation technique affects the number of reproducible features across all of the common modalities used in mPCa imaging, such as CT (60), PET (61), and MRI (62). Open and comprehensive reporting of the technique used is therefore necessary to ensure study reproducibility.

\subsection{Feature Extraction}

The crux of radiomics is the extraction of features from medical images. These features become the basis for which diagnostic and prognostic predictive models are generated which can be utilised to inform clinical decision making that is personalised to the specific biological attributes of the patient. In conventional radiomics practice, mathematically defined features that are hand-crafted using domain knowledge numbering in the hundreds, or sometimes thousands, are extracted from the ROI. However, with the recent surge in deep learning-based models and their applicability to medical images, it has become possible to mitigate the use of hand-crafted features and train complex neural network (NN) and convolutional neural network (CNN) models that are capable of learning the most salient features in unsupervised or supervised manners $(63,64)$. These two distinct types of features extracted from medical images will henceforth be referred to as hand-crafted features and machinelearnt features, respectively.

Hand-crafted features have the ability to capture either spatial or temporal heterogeneities within the defined ROI and can thus be categorised broadly as being either static or dynamic (65). Static radiomic features are time invariant and therefore characterise only spatial properties of the tumour. They are comprised of shape-based (morphological) and statistical features, which are further divided into first-, second-, and higher-order outputs. Morphological features describe the geometry of the lesion such as its compactness, sphericity, or surface to volume ratio $(66,67)$. First-order statistical features are derived from first-order histograms describing the distribution of voxel intensities within the specified tumour volume. Second- 
order statistical features, or what are often referred to as 'texture features', are among the most common descriptors used in radiomics predictive modelling. Texture features are able to characterise intensity spatial interrelationships between tumour voxels that first-order statistics fail to capture (65). Texture features can be extracted from a variety of defined matrices, such as the grey-level co-occurrence matrix (GLCM) (68), the grey-level run length matrix (GLRLM) (69), the grey-level size zone matrix (GLSZM) (70), the neighbourhood grey-tone difference matrix (NGTDM) (71), and the neighbourhood grey-level dependence matrix (NGLDM) (72), for use in predictive modelling. Higher-order statistical features involve the application of various mathematical transformations to the original image from which additional statistical features of firstand second-order can be extracted $(21,73)$, such as Laplacian of gaussian transformations, wavelet decompositions, and gabor filters for edge detection $(74,75)$. Dynamic features capture temporal information about the evolution of the disease over time that can provide more information than a simple snapshot of a lesion at a single time point. These features might unearth new biological characteristics of tumours that can be used in predictive modelling (76). There are, then, a very large array of possible features that can potentially be extracted from the ROI during a radiomics study. In practice, these features need not all be manually defined by the researcher, as the procedure of feature extraction can be performed by a number of commercial and open-source projects dedicated to the task.

Deep NN's and CNN's can be used to automatically learn high-level representations of input data such as medical images and generate machine learnt features. These techniques can be used to perform end-to-end predictive modelling tasks, encompassing automated hierarchical feature extraction and the utilisation of these features for the subsequent classification or regression task in a single step, or alternatively be used as standalone feature extractors (63). Deep CNN's, for example, involve the repeated convolution of learnable filter grids across an input medical image whose values are tuned during the network training process to minimise a cost function such that the salient features relevant to the clinical task at hand are extracted. In this fashion, features are engineered automatically in a hierarchical way where simple characteristics are detected in the lower layers of the network, and increasingly abstract representations of the input image are learned as it progresses deeper through the network architecture $(77,78)$. There is a growing body of evidence demonstrating the usefulness of CNNbased features as a concomitant to traditional hand-crafted features in radiomics studies pertaining to various malignancies, such as soft tissue sarcoma (79), glioma (80), lung cancer (81), and also mPCa (82). Deep learning feature generators have greater versatility by not limiting themselves exclusively to manual human-defined features, however, this comes at the significant cost of reduced interpretability due to the black box nature of these algorithms. The benefits should therefore be weighed against the limitation of model interpretability, which is often desirable in the clinical context (83).

\subsection{Feature Selection}

The dimensions of the extracted feature space may be large relative to the patient sample size, partly due to the nature of medical research where ethical considerations constrain access to patient data. Generating a predictive model with more explanatory variables than patient samples prevents generalisability of the model by over-fitting the sample on which the model was trained $(83,84)$. Reducing the dimensionality of the feature space improves the prediction capabilities of the final model, increases model interpretability, and shortens the training time. Selecting a subset of good features is therefore a crucial part of the radiomics workflow. Feature selection can be conducted according to the calculation of traditional statistical measures where features are eliminated through the application of thresholds, or, the dimensions of the feature space can be reduced by data-driven algorithms that project the data into lower dimensional spaces. Through the conduction of the feature selection process, quantitative imaging biomarkers should be chosen based on the possession of the following properties: repeatability, nonredundancy and reproducibility. Reproducibility has been stressed in the sections above, the main considerations being feature reproducibility with respect to segmentation and scanner variability.

Where possible, available test-retest data should be utilised to assess the repeatability of features across imaging scans taken under identical conditions (85). It is common to select arbitrary thresholds based on, for example, intraclass correlation coefficient (ICC) metrics calculated between the test-retest biomarker outputs, to exclude non-repeatable features $(58,86)$. This simple thresholding method is widely used, but its drawbacks should be noted. Firstly, comparisons of ICC values between different populations are invalid since the metric depends on the variance of the data, and thus also the underlying characteristics of the population under analysis (87, 88). Secondly, repeatability analysis alone is insufficient to determine the usefulness of a quantitative imaging biomarker. In particular, when it comes to response assessment, a feature with low repeatability may change drastically in response to therapy and could therefore be more informative as a predictive biomarker than a feature with high repeatability that changes only minimally during the same treatment. Therefore, it is not appropriate in all cases to remove non-repeatable features based on cut-off values of repeatability metrics. This point has been argued by Lin et al. (89), who posit a new metric for longitudinal assessment of predictive biomarkers termed the 'response-torepeatability' ratio which weighs the biomarker sensitivity (measured as a change between baseline and follow-up scan) against its repeatability.

Redundant features that are highly correlated with each other are unlikely to provide any additional information useful for predictive modelling and can lead to model instability (90). Furthermore, their inclusion can increase the chances of overfitting and hamper model generalisability. Clusters of highly correlated features can be visualised and reduced to a single representative feature that is the most informative in the cluster $(21,87)$. Unsupervised data-driven algorithms that 
project the feature space into a lower dimension can also be used to select non-redundant feature subsets. Principal Component Analysis (PCA), which is a commonly employed method (28, 91, 92 ), is a linear dimensionality reduction technique that identifies successive axes that account for the largest variance in the data and projects the original feature space onto the hyperplane defined by those axes. The new, dimension reduced feature space is comprised of the principal components, each of which is defined by the projection of the original data onto one of the principal axes. The orthogonality of the principal components ensures that feature collinearity is minimised, which makes it an advantageous technique for the unsupervised removal of redundant features (28). Non-linear dimensionality reduction techniques such as local linear embedding (LLE) can also be used for the selection of salient features (84). It should be noted, however, that the projection of features into a lower dimensional space comes at the cost of reduced feature interpretability.

\subsection{Model Development}

Having selected a subset of salient features, the final step in the radiomics workflow is the building of the predictive model. The development of classification models can be done using a variety of ML and deep learning techniques. It is impossible to know $a$ priori which method will generate the best predictive model, and thus experimentation is advised along with comprehensive documentation of the techniques tested, hyperparameters used, and validation results. Scientific reproducibility of radiomics studies is a critical factor that can be facilitated by making implemented code available on platforms such as Github ${ }^{1}$. A summary of some of the more prominent modelling techniques used, and example papers where they have been used in relation to $\mathrm{mPCa}$, are provided in Table $\mathbf{1}$.

\section{CONVENTIONAL MPCa BIOMARKERS AND RISK FACTORS - A BRIEF OVERVIEW}

Various patient biomarkers and characteristics are factored into assessing the risk of developing $\mathrm{mPCa}$ and the clinical management of the disease if it subsequently develops. Patient characteristics such as age, presence of co-morbidities, previous treatment history and personal preference can all affect $\mathrm{mPCa}$ management (101). PSA concentrations carry prognostic information, with elevated levels associated with an increased risk of metastatic development (102). Changes between pre- and post-therapeutic PSA levels is also used to assess patient response to treatment (103), however, the non-specificity of PSA to PCa raises questions about its usefulness in this respect (11). Gleason grading is also a powerful prognostic tool, both for localised PCa and $\mathrm{mPCa}$ in either the castrate-resistant form or the castratesensitive form (104-106). To inform a precision medicine approach to $\mathrm{mPCa}$ management, numerous molecular assays exist that can provide prognostic information. Detecting the

\footnotetext{
${ }^{1}$ https://github.com/
}

presence of the AR-V7 splice variant in circulating tumour cells, for example, is a factor predictive of poor therapeutic response to androgen receptor inhibitors such as enzalutamide and abiraterone (107).

In addition to clinicopathologic characteristics, firstorder radiomics imaging features have also demonstrated usefulness in the clinical management of mPCa. Standardised uptake value $(\mathrm{SUV})_{\max }$ of identified tumours is a prognostic imaging biomarker (108), and when measured from $\left[{ }^{18} \mathrm{~F}\right]$ fluorodeoxyglucose (FDG) PET-avid lesions has been shown to correlate with patient survival (109). Total lesion glycolysis (TLG) or total SUV (SUV total), measured as the sum of individual SUVs in each voxel for each individual lesion, is another prognostic imaging biomarker that has been shown to correlate with overall survival in metastatic castration-resistant prostate cancer (mCRPC) patients (110). Radiomics possesses great potential because rather than attempting to replace these biomarkers, clinicopathologic risk factors and patient characteristics can be incorporated into the modelling process and leveraged to make better model predictions.

\section{REVIEW METHODOLOGY}

\subsection{Study Inclusion Criteria}

Databases were searched using a logical search string [("radiomics" OR "texture analysis" OR "radiological features" OR "radiomic features" OR "textural features" OR "texture features" OR "deep learning” OR "machine learning” OR "convolutional neural network" OR "CNN") AND (metastatic OR metastases) AND ("prostate cancer" OR "prostate lesions")] to identify potentially relevant papers published before the $23^{\text {rd }}$ of June 2021. Inclusion criteria were as follows: (1) human studies only, (2) analysis of medical imaging modalities only (radiomics analyses of histopathology slides were not included), (3) papers must either (i) extract a minimum of second order statistical features, or (ii) use deep learning techniques for feature extraction, (4) results must have diagnostic, prognostic, or predictive applicability to $\mathrm{mPCa},(5)$ minimum of 10 sample size, and (6) full text of article must be available and accessible through our institution. A flow diagram illustrating the inclusion of identified studies is provided in the form of a PRISMA diagram (111) in Figure 2.

The studies were partitioned into two sections following their inclusion into this review, namely, (i) Traditional radiomics, referring to papers that utilised traditional hand-crafted feature extraction techniques, and (ii) Deep radiomics, referring to papers that utilised deep learning networks for the extraction of deep features.

\subsection{RQS Criteria}

Identified papers utilising traditional hand-crafted feature extraction were subject to a RQS analysis. The RQS criteria is comprised of 16 defined items that correspond to critical points in the radiomics workflow. Each item in the RQS either awards or deducts points from a paper according to the study methodology. The RQS is designed to pinpoint methodological 
TABLE 1 | Summary of the prominent modelling techniques used in radiomics research.

\begin{tabular}{|c|c|c|c|c|c|}
\hline Category & $\begin{array}{l}\text { mPCa } \\
\text { Papers }\end{array}$ & Technique & Description & Advantages & Disadvantages \\
\hline \multirow[t]{5}{*}{$\begin{array}{l}\text { Machine } \\
\text { Learning }\end{array}$} & (93) & Support Vector Machine & $\begin{array}{l}\text { Determines the support vectors in } \\
\text { multidimensional space that define the } \\
\text { hyperplane best separating the classes }\end{array}$ & $\begin{array}{l}\text { Versatile algorithm capable of } \\
\text { both linear and non-linear } \\
\text { classification }\end{array}$ & $\begin{array}{l}\text { Not suited to large datasets } \\
\text { Model is difficult to interpret } \\
\text { Sensitive to feature scaling }\end{array}$ \\
\hline & $\begin{array}{l}(94 \\
95)\end{array}$ & Logistic Regression & Binary classification algorithm & $\begin{array}{l}\text { Probabilistic output } \\
\text { Relatively quick training time }\end{array}$ & $\begin{array}{l}\text { Linear assumption makes it } \\
\text { unsuitable for highly complex } \\
\text { datasets }\end{array}$ \\
\hline & (96) & K- Nearest Neighbour & $\begin{array}{l}\text { Classifies new instances based on their } \\
\text { calculated distance from the k-nearest } \\
\text { neighbours in the memorised training set. }\end{array}$ & $\begin{array}{l}\text { Instance-based model } \\
- \text { no training period }\end{array}$ & $\begin{array}{l}\text { Does not scale well to large } \\
\text { datasets } \\
\text { Sensitive to noise, outliers and } \\
\text { feature scale }\end{array}$ \\
\hline & (96) & Decision Tree & $\begin{array}{l}\text { Hierarchical model structure resembling a tree } \\
\text { where instances are partitioned at nodes } \\
\text { based on feature threshold values until a final } \\
\text { classification is made at the leaf nodes }\end{array}$ & $\begin{array}{l}\text { Highly interpretable - 'white } \\
\text { box' classification method } \\
\text { Non-parametric model } \\
\text { - makes limited assumptions } \\
\text { about training data } \\
\text { Insensitive to feature scale }\end{array}$ & $\begin{array}{l}\text { Prone to overfitting } \\
\text { Small changes in training data } \\
\text { can cause large changes to } \\
\text { tree structure, leading to mode } \\
\text { instability. }\end{array}$ \\
\hline & $\begin{array}{l}(97 \\
98)\end{array}$ & $\begin{array}{l}\text { Ensemble-Based Methods } \\
\text { (Random Forest, Stacked } \\
\text { Generalisation, } \\
\text { Bootstrapping } \\
\text { Aggregation, etc.) }\end{array}$ & $\begin{array}{l}\text { Aggregates predictions from multiple trained } \\
\text { classifiers and combines them into a single } \\
\text { predictive model }\end{array}$ & $\begin{array}{l}\text { Can combine several weak } \\
\text { classifiers into a single strong } \\
\text { classifier } \\
\text { Reduces prediction model bias }\end{array}$ & $\begin{array}{l}\text { Very limited model interpretability } \\
\text { Depending on the base classifiers } \\
\text { used, extensive } \\
\text { hyperparameter tuning may be } \\
\text { required. }\end{array}$ \\
\hline \multirow[t]{2}{*}{$\begin{array}{l}\text { Deep } \\
\text { Learning }\end{array}$} & (99) & $\begin{array}{l}\text { Convolutional Neural } \\
\text { Network }\end{array}$ & $\begin{array}{l}\text { Repeated application of convolution } \\
\text { operations to an input vector/image to learn } \\
\text { salient feature maps for the desired } \\
\text { classification task. }\end{array}$ & $\begin{array}{l}\text { Capable of performing end-to- } \\
\text { end feature extraction and } \\
\text { predictive modelling } \\
\text { Highly versatile } \\
\text { Automatically learns important } \\
\text { image features without the } \\
\text { need for hand-crafted feature } \\
\text { engineering }\end{array}$ & $\begin{array}{l}\text { Potentially limitless number of } \\
\text { possible architectures with } \\
\text { limited a priori knowledge of } \\
\text { which is best suited to the task } \\
\text { Typically require large datasets to } \\
\text { be generalisable } \\
\text { Large training time } \\
\text { Severely limited model } \\
\text { interpretability }\end{array}$ \\
\hline & $\begin{array}{r}(82 \\
100)\end{array}$ & Transfer Learning & $\begin{array}{l}\text { Extracting layers in deep networks that have } \\
\text { been pre-trained on large datasets to be used } \\
\text { as feature extractors for your specific task }\end{array}$ & $\begin{array}{l}\text { Useful for small dataset } \\
\text { problems where there are } \\
\text { insufficient samples to train a } \\
\text { full deep network } \\
\text { Can decrease training time } \\
\text { significantly }\end{array}$ & $\begin{array}{l}\text { Can fail if the outcome of the pre- } \\
\text { trained network differs } \\
\text { significantly from that of the } \\
\text { target outcome. } \\
\text { Severely limited model } \\
\text { interpretability }\end{array}$ \\
\hline
\end{tabular}

Exemplar papers using each of these in MPCa radiomics studies are provided.

weaknesses in radiomics studies and encourage scientifically rigorous radiomics investigations (32). Table 2 provides a full description of these items and the points that can potentially be gained at each. A study may achieve a maximum of 36 points, or a minimum of -8 points. Each paper was assigned a percentage based on their score out of 36 .

\section{RADIOMICS IN MPCa - RESULTS}

\subsection{Traditional Radiomics}

The use of the traditional radiomics paradigm of utilising handcrafted features in $\mathrm{mPCa}$ is the predominant method of solving clinical challenges. Tables 3, 4 summarise the salient characteristics for all identified traditional radiomics papers.

\subsubsection{Metastasis Prediction and Detection}

The prediction of metastases development, or the detection of its presence, are the most common outcome objectives of the traditional radiomics studies identified in this review. Wang et al. (94) constructed a prognostic model for the pretreatment prediction of bone metastases in patients with histologically confirmed PCa without evidence of distant metastatic spread. 976 radiomic features, including first-order statistics, shape features and higher order texture features, were extracted from the outlined primary PCa lesion on T2-weighted (T2w) and dynamic contrast-enhanced (DCE) T1-weighted (T1w) images. The final logistic regression model, comprised of imaging features weighted by their least absolute shrinkage and selection operator (LASSO) regression coefficients and clinicopathologic patient variables (age, GS, free PSA) achieved good discriminative performance in predicting future bone metastases in the internal validation cohort, with an AUC of 0.895 (95\% CI 0.836 - 0.939). The radiomics model outperformed traditional prognostication methods, such as the GS (AUC = 0.731), demonstrating the potential for non-invasive assessment of primary PCa tumours on mp-MRI for prediction of future bone metastases, with potentially profound impacts for 


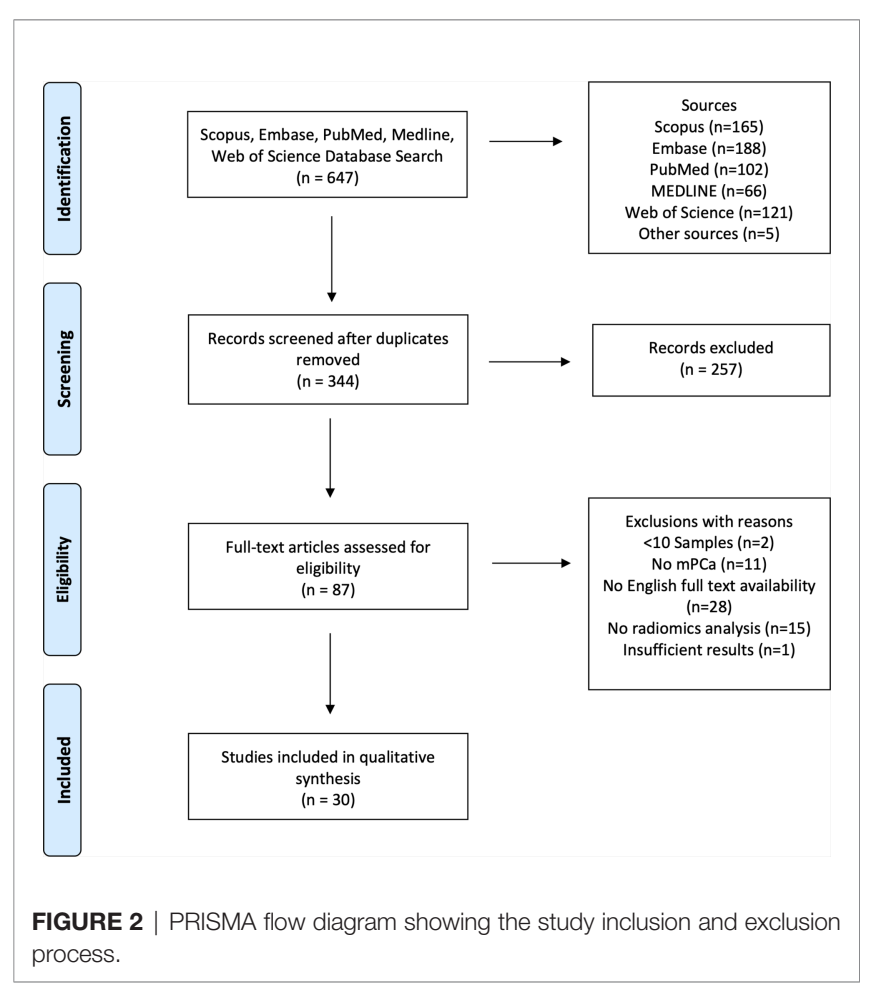

individualised patient therapeutic decision making. These results are in good agreement with those achieved by Zhang and colleagues (95) who developed a combined radiomics and clinicopathologic variable (total PSA) nomogram for assessing the risk of bone metastases in newly diagnosed PCa patients from features extracted from T2w, DCE T1w and diffusionweighted imaging (DWI) scans. LASSO regression was used to perform feature selection and generate the radiomics score, where each feature was weighted by its regression coefficient, and then combined with the total PSA clinical variable in a multivariable logistic regression analysis. The final nomogram achieved good predictive performance in an internal validation cohort $(\mathrm{n}=35)$ in the prediction of developing bone metastases with an AUC $=0.93$ (95\% CI, $0.86-0.99)$.

PET imaging features can be highly effective in the detection of PCa metastases. Cysouw et al. (97) conducted a study on a cohort of 76 intermediate- to high-risk primary PCa patients who were scheduled to undergo radical prostatectomy, where radiomics features were extracted from the delineated intraprostatic tumour volume to develop a diagnostic model for detecting the presence of either lymph node metastases or any distant metastases in $\left[{ }^{18} \mathrm{~F}\right] \mathrm{DCFPyL}$ PET scans. Intensity, shapebased and texture features were extracted from the delineated intraprostatic primary PCa lesion and subject to ML analysis. The resulting random forest algorithm trained on a subset of features chosen via three different feature selection methods (PCA, recursive feature elimination with random forest, and univariate analysis of variance) and utilising 5-fold cross validation achieved good discriminatory performance in the detection of LNI (AUC $=0.86 \pm 0.15, p<0.01$ ), and any distant metastasis (AUC $=0.86 \pm 0.14, p<0.01$ ). Feature importance analysis of the random forest $\mathrm{ML}$ algorithm

TABLE 2 | Details of RQS items and associated points awarded/deducted at each step.

\begin{tabular}{|c|c|c|}
\hline RQS Item & RQS Item Name & Item Description (points awarded/subtracted) \\
\hline 1 & Image protocol quality & $\begin{array}{l}\text { Well documented image protocols }(+1) \\
\text { Public protocol used }(+1)\end{array}$ \\
\hline 2 & Multiple Segmentations & Feature robustness testing to segmentation variabilities, e.g. different algorithms or physicians $(+1)$ \\
\hline 3 & Phantom study on all scanners & Feature robustness testing to scanner parameters $(+1)$ \\
\hline 4 & Multiple time points & Feature robustness testing to temporal variabilities e.g. organ movement/shrinkage $(+1)$ \\
\hline 5 & Feature reduction & Perform feature reduction or adjust for multiple testing (+3); otherwise $(-3)$ \\
\hline 6 & Non-radiomics feature inclusion & Model includes non-radiomic variables/features $(+1)$ \\
\hline 7 & Detect biological correlates & Detect and discuss biological correlates $(+1)$ \\
\hline 8 & Cut-off analysis & Determine risk groups by either the median, a previously published cut-off value or present a continuous risk variable $(+1)$ \\
\hline 9 & Discrimination statistics & $\begin{array}{l}\text { Report a discrimination statistic and its statistical significance }(+1) \\
\text { A resampling method is also applied }(+1)\end{array}$ \\
\hline 10 & Calibration statistics & $\begin{array}{l}\text { Report a calibration statistic and its statistical significance }(+1) \\
\text { A resampling method is also applied }(+1)\end{array}$ \\
\hline 11 & Prospective study & Prospective methodology to validate a radiomics signature $(+7)$ \\
\hline 12 & Validation & $\begin{array}{l}\text { No validation }(-5) \\
\text { Internal validation }(+2) \\
\text { External validation from one institute }(+3) \\
\text { External validation from two institutes }(+4) \\
\text { Validating a previously published radiomics signature }(+4) \\
\text { External validation from three or more institutes }(+5)\end{array}$ \\
\hline 13 & Gold standard comparison & Assess model agreement/superiority to current 'gold standard' (+2) \\
\hline 14 & Clinical utility & Quantify model applicability in clinical setting e.g. decision curve analysis (+2) \\
\hline 15 & Cost-effectiveness assessment & Assess cost-effectiveness of radiomics signature $(+2)$ \\
\hline 16 & Open science and data & $\begin{array}{l}\text { Scans are open source }(+1) \\
\text { Region of interest segmentations are open source }(+1) \\
\text { Code is open source }(+1) \\
\text { Features are calculated on an open-source set of representative features }(+1)\end{array}$ \\
\hline
\end{tabular}


TABLE 3 | Prominent characteristics of identified mPCa studies using traditional radiomics methods with PET and/or CT modalities.

\begin{tabular}{|c|c|c|c|c|c|c|c|c|c|c|c|}
\hline $\begin{array}{l}\text { First } \\
\text { Author }\end{array}$ & Year & $\begin{array}{l}\text { Imaging } \\
\text { Series } \\
\text { Analysed }\end{array}$ & Design & $\begin{array}{l}\text { Patient } \\
\text { sample } \\
\text { size }\end{array}$ & Patient Cohort & $\begin{array}{l}\text { Radiomics } \\
\text { Software }\end{array}$ & $\begin{array}{l}\text { Outcome } \\
\text { Measures }\end{array}$ & $\begin{array}{l}\text { Clinical } \\
\text { Covariates } \\
\text { Included }\end{array}$ & $\begin{array}{c}\text { External } \\
\text { Validation }\end{array}$ & Results Synopsis & $\begin{array}{l}\text { RQS } \\
(\%)\end{array}$ \\
\hline Acar E (96) & 2019 & CT & $\mathrm{R}$ & 75 & $\begin{array}{l}\text { PCa patients with } \\
\text { metastatic bone } \\
\text { disease }\end{array}$ & LifeX & $\begin{array}{l}\text { Sclerotic } \\
\text { Bone Lesion } \\
\text { Response }\end{array}$ & $\mathrm{N}$ & $\mathrm{N}$ & $\begin{array}{l}\text { Acc }=73.5 \% \\
\text { AUC }=76.0 \% \\
\text { Sens }=73.5 \% \\
\text { Spec }=73.7 \%\end{array}$ & 0 \\
\hline $\begin{array}{l}\text { Alongi P } \\
(112)\end{array}$ & 2021 & $\begin{array}{l}{ }^{18} \mathrm{~F}- \\
\text { Choline } \\
\text { PET }\end{array}$ & $\mathrm{R}$ & 94 & High Risk PCa & LifeX & $\begin{array}{l}\text { LNI, Distant } \\
\text { Metastasis }\end{array}$ & Y & $\mathrm{N}$ & $\begin{array}{l}\frac{\mathrm{LNI}}{\mathrm{AUC}=69.87(95 \% \mathrm{Cl}} \\
51.34-88.39) \\
\text { Distant Metastasis } \\
\mathrm{AUC}=74.72(95 \% \mathrm{Cl} \\
56.36-93.09)\end{array}$ & 27.78 \\
\hline $\begin{array}{l}\text { Cysouw } \\
\text { M.C.F (97) }\end{array}$ & 2020 & $\begin{array}{l}{ }^{18} \mathrm{~F}- \\
\text { DCFPyL } \\
\text { PET }\end{array}$ & $\mathrm{P}$ & 76 & $\begin{array}{l}\text { Intermediate- to } \\
\text { high-risk primary } \\
\text { PCa }\end{array}$ & RaCaT & $\begin{array}{l}\text { LNI, Distant } \\
\text { Metastasis }\end{array}$ & $N$ & $\mathrm{~N}$ & $\begin{array}{l}\text { LNI } \\
\text { AUC }=0.86 \pm 0.15 \\
p<0.01 \\
\text { Distant Metastasis } \\
\text { AUC }=0.86 \pm 0.14 \\
p<0.01\end{array}$ & 47.22 \\
\hline $\begin{array}{l}\text { Hayakawa } \\
\text { T (113) }\end{array}$ & 2020 & $\mathrm{CT}$ & $\mathrm{R}$ & 69 & $\begin{array}{l}\text { PCa patients with } \\
\text { pelvic bone } \\
\text { metastases }\end{array}$ & 3D Slicer & os, CSS & $Y$ & $\mathrm{~N}$ & $\begin{array}{l}\text { Maximum 2D Diameter and } \\
\text { Least Axis were risk factors } \\
\text { for OS (HR }=1.007 \& \mathrm{HR}= \\
1.013 \text {, respectively) } \\
\text { No features were risk } \\
\text { factors of CSS. }\end{array}$ & 0 \\
\hline $\begin{array}{l}\text { Khurshid Z } \\
\text { (103) }\end{array}$ & 2018 & $\begin{array}{l}{ }^{68} \mathrm{Ga}- \\
\text { PSMA } \\
\text { PET }\end{array}$ & $\mathrm{R}$ & 70 & $\begin{array}{l}\text { mCRPC patients } \\
\text { planned to } \\
\text { undergo }{ }^{177} \text { Lu- } \\
\text { PSMA therapy }\end{array}$ & NS & $\begin{array}{l}\text { Correlation } \\
\text { with } \\
\text { treatment } \\
\text { response }\end{array}$ & $\mathrm{N}$ & $\mathrm{N}$ & $\begin{array}{l}\text { Entropy and Homogeneity } \\
\text { correlated with response } \\
\text { (r=-0.327 \& } r=0.315 \\
\text { respectively) }\end{array}$ & 8.33 \\
\hline Lin C (89) & 2019 & $\begin{array}{l}{ }^{18} \mathrm{~F}-\mathrm{NaF} \\
\text { PET }\end{array}$ & $\mathrm{P}$ & 14 & $\begin{array}{l}\text { mCRPC patients } \\
\text { undergoing } \\
\text { androgen therapy. }\end{array}$ & NS & $\mathrm{R} / \mathrm{R}$ & $\mathrm{N}$ & $\mathrm{N}$ & $\begin{array}{l}\text { Skewness, Kurtosis and } \\
\text { diagonal moment exhibited } \\
\text { greater R/R than SUV } \max \end{array}$ & 5.56 \\
\hline $\begin{array}{l}\text { Moazemi S } \\
(114)\end{array}$ & 2020 & $\begin{array}{l}{ }^{68} \mathrm{Ga}- \\
\mathrm{PSMA} \\
\text { PET/CT }\end{array}$ & $\mathrm{R}$ & 72 & $\begin{array}{l}\text { Histologically } \\
\text { confirmed PCa }\end{array}$ & $\begin{array}{l}\text { InterView } \\
\text { FUSION } \\
\text { Software }\end{array}$ & $\begin{array}{l}\text { Hotspot } \\
\text { classification }\end{array}$ & $\mathrm{N}$ & $\mathrm{N}$ & $\begin{array}{l}\mathrm{AUC}=0.98, \text { Sens }=0.94 \\
\mathrm{Spec}=0.89\end{array}$ & 5.56 \\
\hline $\begin{array}{l}\text { Moazemi S } \\
(115)\end{array}$ & 2021 & $\begin{array}{l}{ }^{68} \mathrm{Ga}- \\
\mathrm{PSMA} \\
\text { PET/CT }\end{array}$ & $\mathrm{R}$ & 83 & $\begin{array}{l}\text { Advanced PCa } \\
\text { undergoing }{ }^{177} \mathrm{Lu}- \\
\text { PSMA therapy. }\end{array}$ & NS & OS & Y & $\mathrm{N}$ & $\begin{array}{l}\text { PET Kurtosis \& SUV } V_{\min } \\
\text { significantly correlated with } \\
\text { OS }\end{array}$ & 0 \\
\hline $\begin{array}{l}\text { Peeken J } \\
(116)\end{array}$ & 2020 & $\mathrm{CT}$ & $\mathrm{R}$ & 80 & Recurrent PCa & PyRadiomics & LNI & $\mathrm{N}$ & Y & $\begin{array}{l}\mathrm{AUC}=0.95(95 \% \mathrm{Cl} 0.88- \\
0.99)\end{array}$ & 47.22 \\
\hline Perk T (98) & 2018 & $\begin{array}{l}{ }^{18} \mathrm{~F}-\mathrm{NaF} \\
\mathrm{PET} / \mathrm{CT}\end{array}$ & $\mathrm{R}$ & 37 & mCRPC patients & NS & $\begin{array}{l}\text { Bone Lesion } \\
\text { Classification }\end{array}$ & Y & $\mathrm{N}$ & $\begin{array}{l}A \cup C=0.95(95 \% \mathrm{Cl} 0.93- \\
0.96)\end{array}$ & 25 \\
\hline $\begin{array}{l}\text { Zamboglou } \\
\text { C (117) }\end{array}$ & 2019 & $\begin{array}{l}{ }^{68} \mathrm{Ga}- \\
\text { PSMA } \\
\text { PET }\end{array}$ & Both & $\begin{array}{l}P(n=20) \\
R(n=40)\end{array}$ & $\begin{array}{l}\text { Histopathologically } \\
\text { proven primary } \\
\text { adenocarcinoma }\end{array}$ & $\begin{array}{l}\text { In-house } \\
\text { MATLAB } \\
\text { software }\end{array}$ & $\mathrm{LNI}$ & $\mathrm{N}$ & $\mathrm{N}$ & $\begin{array}{l}\text { Prospective Cohort } \\
\text { AUC }=0.87 \\
\text { Retrospective Cohort } \\
\text { AUC }=0.85\end{array}$ & 52.78 \\
\hline
\end{tabular}


repeatability; Sens, Sensitivity; Spec, Specificity.

determined that two intensity-based features, difference volume at intensity fraction and volume at intensity fraction 10, were the most important in detecting LNI and distant metastases (both had importance coefficients of 0.11 ). The clinical relevance of the ML model is that it can non-invasively aid in the determination of low-risk patients that can be spared extended pelvic lymph node dissection and its associated complications (120).

A similar study was conducted by Zamboglou et al. (117), who enrolled both a prospective $(n=20)$ and a retrospective $(n=40)$ validation cohort of patients with intermediate- to high-risk PCa who underwent ${ }^{68} \mathrm{Ga}$-PSMA PET/CT imaging prior to RP. Intraprostatic lesions in the PSMA PET image in the prospective cohort were segmented in three ways; (i) by histopathologic analysis and ex-vivo CT scanning of the RP specimen, which was subsequently transferred to the in-vivo CT scan before being transformed into the PSMA PET coordinates; (ii) manually by two nuclear medicine physicians, and; (iii) semi-automatically by the application of local $40 \%$ thresholds in the intraprostatic volume. The retrospective validation cohort was segmented only manually. In the prospective cohort, the QSZHGE (quantised short zones highgray level emphasis), which was robust to different scanner parameters (determined in a phantom study) and to the three segmentation methods, was able to discriminate well between patients with and without LNI (AUC $=0.87$ for the expert manual segmentation, and AUC $=0.85$ for the histopathologic 
TABLE 4 | Prominent characteristics of identified mPCa studies using traditional radiomics methods with MRI modalities.

\begin{tabular}{|c|c|c|c|c|c|c|c|c|c|c|c|}
\hline $\begin{array}{l}\text { First } \\
\text { Author }\end{array}$ & Year & $\begin{array}{l}\text { Imaging } \\
\text { Series } \\
\text { Analysed }\end{array}$ & Design & $\begin{array}{l}\text { Patient } \\
\text { sample } \\
\text { size }\end{array}$ & $\begin{array}{l}\text { Patient } \\
\text { Cohort }\end{array}$ & $\begin{array}{l}\text { Radiomics } \\
\text { Software }\end{array}$ & $\begin{array}{l}\text { Outcome } \\
\text { Measures }\end{array}$ & $\begin{array}{l}\text { Clinical } \\
\text { Covariates } \\
\text { Included }\end{array}$ & $\begin{array}{c}\text { External } \\
\text { Validation }\end{array}$ & Results Synopsis & $\begin{array}{c}\text { RQS } \\
(\%)\end{array}$ \\
\hline $\begin{array}{l}\text { Damascelli } \\
\text { A (93) }\end{array}$ & 2021 & $\begin{array}{l}\mathrm{T} 2 \mathrm{w} \\
\mathrm{ADC}\end{array}$ & $\mathrm{R}$ & 62 & $\begin{array}{l}\text { Biopsy } \\
\text { confirmed } \\
\text { PCa who } \\
\text { underwent RP }\end{array}$ & 3D Slicer & LNI & $\mathrm{N}$ & $N$ & $\begin{array}{l}\text { Acc }=0.9 \pm 0.04 \\
\text { Sens }=0.9 \pm 0.01 \\
\text { Spec }=0.9 \pm 0.01\end{array}$ & 25 \\
\hline Hou Y (82) & 2021 & $\begin{array}{l}\text { T2W, } \\
\text { DWI, } \\
\text { ADC }\end{array}$ & $\mathrm{R}$ & 401 & $\begin{array}{l}\text { Biopsy } \\
\text { confirmed } \\
\text { PCa patients } \\
\text { who } \\
\text { underwent RP } \\
\text { \& ePLND }\end{array}$ & PyRadiomics & $\begin{array}{l}\text { Pelvic } \\
\text { Lymph } \\
\text { Node } \\
\text { Metastases }\end{array}$ & Y & Y & $\mathrm{AUC}=0.76(95 \% \mathrm{Cl}, 0.62-0.87)$ & 33.33 \\
\hline Li L (118) & 2021 & $\begin{array}{l}\mathrm{T} 2 \mathrm{w} \\
\mathrm{ADC}\end{array}$ & $\mathrm{R}$ & 198 & $\begin{array}{l}\text { PCa patients } \\
\text { who } \\
\text { underwent RP }\end{array}$ & NS & $\mathrm{LNI}$ & Y & Y & $\mathrm{AUC}=0.77(95 \% \mathrm{Cl}, 0.67-0.86)$ & 50 \\
\hline $\begin{array}{l}\text { Reischauer } \\
\text { C (119) }\end{array}$ & 2018 & ADC & $\mathrm{P}$ & 12 & $\begin{array}{l}\text { Treatment- } \\
\text { naïve } \\
\text { advanced PCa } \\
\text { with } \\
\text { scintigram } \\
\text { confirmed } \\
\text { metastases }\end{array}$ & $\begin{array}{l}\text { In-house } \\
\text { MATLAB } \\
\text { software }\end{array}$ & $\begin{array}{l}\text { Change in } \\
\text { response } \\
\text { to ADT } \\
\text { therapy }\end{array}$ & $\mathrm{N}$ & $\mathrm{N}$ & $\begin{array}{l}\text { Numerous first- and second-order } \\
\text { statistical features showed } \\
\text { promise in monitoring treatment } \\
\text { response and correlation to } \\
\text { changes in serum PSA levels over } \\
\text { time. }\end{array}$ & 0 \\
\hline $\begin{array}{l}\text { Wang } Y \\
(94)\end{array}$ & 2019 & $\begin{array}{l}\text { T2w, } \\
\text { DCE T1w }\end{array}$ & $\mathrm{R}$ & 176 & $\begin{array}{l}\text { Histologically } \\
\text { confirmed } \\
\text { PCa without } \\
\text { evidence of } \\
\text { distant } \\
\text { metastasis }\end{array}$ & IBEX & $\begin{array}{l}\text { Prediction } \\
\text { of Bone } \\
\text { Metastasis }\end{array}$ & Y & $\mathrm{N}$ & $\begin{array}{l}A \cup C=0.895(95 \% \mathrm{Cl} 0.836- \\
0.939)\end{array}$ & 25 \\
\hline $\begin{array}{l}\text { Zhang W } \\
\text { (95) }\end{array}$ & 2020 & $\begin{array}{l}\text { T2w, } \\
\text { DCE } \\
\text { T1w, DWI }\end{array}$ & $\mathrm{R}$ & 116 & $\begin{array}{l}\text { Biopsy } \\
\text { confirmed } \\
\text { PCa }\end{array}$ & AK Software & $\begin{array}{l}\text { Bone } \\
\text { Metastases }\end{array}$ & Y & $\mathrm{N}$ & $A \cup C=0.93(95 \% \mathrm{Cl}, 0.86-0.99)$ & 38.89 \\
\hline
\end{tabular}

Acc, Accuracy; ADT, Androgen deprivation therapy; AUC, Area Under Curve; ePLND, extended pelvic lymph node dissection; NS, Not Specified; OS, Overall Survival; P, Prospective; R, Retrospective; Sens, Sensitivity; Spec, Specificity.

segmentation, both $p<0.01)$. This performance was validated in the retrospective cohort (AUC $=0.85, p<0.01$ ). Alongi et al. (112) analysed 94 high-risk PCa patients who underwent ${ }^{18} \mathrm{~F}$ Choline PET/CT restaging imaging to determine features predictive of disease progression. Follow up data was recorded for the patients for a median of 26 months (range 13-52) and subsequent TNM staging performed. Discriminant analysis on the radiomics features extracted yielded a machine learning (ML) model capable of achieving moderate predictive power in the development of nodal (AUC $=69.87,95 \%$ CI 51.34-88.39) or distant metastases (AUC $=74.72,95 \%$ CI 56.36-93.09). HISTO entropy $\log 10$ and HISTO entropy $\log 2$ were the two salient features chosen for the discrimination of distant metastases, while GLSZM SZLGE and HISTO energy uniformity were the chosen features to predict nodal metastases.

Studies have also shown the great potential of radiomics features extracted from mpMRI modalities in the detection and prediction of metastases. Damascelli et al. (93) developed a radiomics model for the prediction of LNI on 62 patient mpMRI scans, where features were extracted from T2w images and apparent diffusion coefficient (ADC) maps. Each patient, who had biopsy proven PCa and underwent RP, had their intraprostatic index lesions segmented on each modality by two independent radiologists. Features not robust to the two segmentations (Friedman test $p$ value $>0.01$ ) were excluded, and the covariance matrix analysed to remove redundant features. Support vector machine classifiers were built using features from each modality both separately and in combination, and were able to predict lymph node metastasis presence with good accuracy $(\mathrm{ADC}$ alone, $\mathrm{Acc}=0.86 \pm 0.05 ; \mathrm{T} 2 \mathrm{w}$ alone, $\mathrm{Acc}=0.84 \pm 0.05$; $\mathrm{ADC}+\mathrm{T} 2 \mathrm{w}$ features, $\mathrm{Acc}=0.9 \pm 0.04)$. Their results demonstrate the promise of utilising radiomics signatures derived from mpMRI scans to predict LNI and the potential of utilising features extracted from multiple modalities in radiomics predictive modelling to capture complementary information about lesions and improve overall model performance. Li et al. (118) performed a comparable analysis and developed a prognostic radiomics nomogram for the pre-operative prediction of lymph node metastases also in PCa patients who underwent RP. A total of 200 radiomic features were extracted from the intraprostatic index lesion delineated in both the $\mathrm{T} 2 \mathrm{w}$ image and the ADC maps, where non-repeatable features were excluded by performing a test-retest analysis on an openly available test-retest mpMRI dataset (121), and the remaining features were selected using a 5-fold 10-run cross validation of a minimum redundancy maximum relevance algorithm for Coxproportional hazards model building. This model was combined with clinicopathologic patient data (PSA and GS) to generate the final prognostic nomogram, which was externally validated on a multi-institutional dataset. Validation set performance in the 
prediction of LNI was AUC $=0.77$ (95\% CI, 0.67-0.86), and this result compared favourably to other prognostic tools used for the prediction of post-treatment adverse pathology such as the Prostate Cancer Risk Assessment score (AUC $=0.74 ; 95 \%$ CI, $0.62-0.85$ ) and the Decipher risk score (AUC $=0.73$; 95\% CI, $0.59-0.87)$. The multi-institutional validation and superior performance of this nomogram compared to other prognostic tools is strong evidence for the potential of utilising radiomics features for the pre-operative risk stratification of patients.

Hou et al. (82) in a recent study explored how traditional radiomics features can be supplemented with deep learning machine learnt features in the prediction of pelvic lymph node metastases (PLNMs). A relatively large sample size of 401 patients (including a 50-patient external validation set) with biopsy confirmed PCa were analysed, where 2553 radiomics features were extracted in total from the index intraprostatic lesion on mpMRI modalities (T2w, DWI with $b=1500 \mathrm{~s} / \mathrm{mm}^{2}$ and ADC maps) to generate a radiomics signature. In parallel, five pre-trained deep neural networks trained on ImageNet data were utilised in a transfer learning framework which leverages the high-level salient feature extraction ability of the pre-trained networks and applies them to the present problem. Random forest algorithms were used to output a final risk score reflecting the probability of the patient developing pelvic lymph node metastatic disease for both the radiomics and the deep learning signatures combined and individually. The final risk model yielded an AUC $=0.76(95 \% \mathrm{CI}, 0.62-0.87)$ on the external hold-out set. Furthermore, the authors compared their risk model with established prognostic tools such as the Memorial Sloan Kettering Cancer Center (MSKCC) $)^{2}$ nomogram and the Briganti score (122), determining that an optimal threshold of $8 \%$ on their risk model is superior to both in terms of sparing unnecessary pelvic lymph node dissections and missing fewer true positive PLNMs.

\subsubsection{Lesion Classification}

Radiomics features have also been used successfully to classify the malignancy of segmented lesions, for example, Peeken et al. (116) developed and externally validated a radiomics signature extracted from contrast-enhanced CT images to determine the malignancy (positive or negative) of segmented lymph nodes. A total of 149 lymph nodes were segmented from which shape, first-order, textural, and local binary pattern-based intensity features were extracted. LASSO regression was used to select salient features for the final model, which performed well on a dedicated external validation cohort ( $\mathrm{n}=33$ patients) with an AUC $=0.95$ (95\% CI $0.88-0.99)$. Their results demonstrate that radiomics feature extraction can be extended to ROIs other than the tumour volume, such as segmented lymph nodes, and still yield accurate predictive models. Additionally, the authors of this study also utilised ComBat batch harmonisation to correct for the difference in scanner parameters between the external validation cohort and the main training cohort, but found that the use of this technique did not significantly change the results of the radiomics model.

$\overline{{ }^{2} \text { https://www.mskcc.org/nomograms/prostate }}$
Similar lesion classification analysis has been conducted on other imaging modalities. Moazemi et al. (114) employed ML analysis on radiomics features to classify $2419{ }^{68} \mathrm{Ga}$-PSMA PET hotspots (defined as uptake above the background concentration) in 72 patients as either benign or malignant. A total of 80 features were extracted from each hotspot (40 from the PET image and 40 from the CT image) and utilised in five different ML algorithms, with the Extra Trees model achieving exemplary discriminatory performance in the classification of lesions in the hold-out validation set $(\mathrm{n}=24)$ with $\mathrm{AUC}=0.98$ (Sens $=94 \%$, Spec $=$ $89 \%)$. Their results also further demonstrate the validity of multimodal analysis since features from the CT and PET images used together in the ML models slightly outperforms using either of them separately. Perk et al. (98) conducted a similar analysis on ${ }^{18} \mathrm{~F}-\mathrm{NaF}$ PET/CT images in a cohort of $37 \mathrm{mCRPC}$ subjects. Bone lesions were delineated by an automated algorithm that determines the lesion boundaries based on statistically optimised regional thresholding (SORT) which uses a different threshold based on the location of the lesion in the patients skeleton (123), which were subsequently assigned a classification label by a nuclear medicine physician between 0 and 5 depending on the likelihood of malignancy (0; background ROI, 1; Definite Benign, 5; Definite Malignant). Radiomics features were extracted from both the PET and CT images and used as the input for ML analysis with nine separate learning methods, where the random forest model performed the best under 10 -fold cross-validation conditions at discriminating between the $0+1$ vs. 5 class labels $(\mathrm{AUC}=0.95,95 \%$ CI $0.93-0.96)$.

\subsubsection{Treatment Response}

PSMA-labelled isotopes are becoming increasingly important in the imaging and treatment of metastatic PCa. ${ }^{177} \mathrm{Lu}$-PSMA therapy, in particular, is gaining prominence as a radioligand therapeutic intervention for advanced PCa, however, it is known that a large percentage of interventions will not be successful (124). Early identification of which patients may benefit from a particular intervention type can be substantially aided by radiomics analysis which can yield useful pre-therapeutic biomarkers. Moazemi et al. (115) extracted radiomics features from delineated hotspots $(n=2070)$ in advanced PCa patients with pre-therapeutic ${ }^{68} \mathrm{Ga}$-PSMA PET/CT imaging. Following a LASSO regression feature selection process, they determined that a radiomics signature containing PET Kurtosis and $S U V_{\min }$ were significantly correlated with overall survival $(r=0.2765$, $p=0.0114$ ). An earlier study by Khurshid et al. (103) performed a similar retrospective analysis on $70 \mathrm{mCRPC}$ patients scheduled to undergo ${ }^{177} \mathrm{Lu}$-PSMA therapy. Metastatic lesions in each patient (total ROI's $=328$ ) were segmented, from which histogram and textural features from the normalised gray-level co-occurrence matrix (NGLCM) were extracted. Therapeutic response was defined according to the change in pre- and posttherapy PSA levels, which is common practice, although, as an aside, recent evidence has demonstrated the potential for texture features to be used as biomarkers for therapeutic response assessment (119). Their analysis showed that two textural parameters extracted from the NGLCM of bone lesions correlated with the change in PSA levels following therapy, 
these being entropy $(r=-0.327, p=0.006)$ and homogeneity $(r=$ $0.315, p<0.008)$. The results therefore indicated that the lesions which were more heterogeneous in nature responded better to the ${ }^{177} \mathrm{Lu}$-PSMA therapy. This is an interesting result that can potentially inform future clinical decision making regarding the use of ${ }^{177} \mathrm{Lu}-\mathrm{PSMA}$ radioligand therapy, pending future prospective and external validation.

Determining the status of sclerotic metastatic lesions posttreatment is difficult because even responded lesions that are no longer metastatic can retain their sclerotic character when pretreatment and post-treatment CT images are compared. Acar et al. (96) sought to utilise radiomics imaging features to discriminate between sclerotic lesions that were completely responded or still metastatic following therapeutic interventions. Histogram, shape-based, and texture features were extracted from manually delineated sclerotic bone lesions in the non-contrast CT scan of each patient. Sclerotic lesions were categorised as completely responded or metastatic if they had ${ }^{68} \mathrm{Ga}$-PSMA PET uptake levels either below or above the measured liver expression. Multiple ML models were developed, including a weighted K-nearest neighbour (KNN), support vector machine, decision tree and ensemble-based methods, where the weighted KNN achieved the best classification performance under 10 -fold cross-validation conditions (Accuracy $=73.5 \%$, AUC $=76.0 \%$, Sensitivity $=73.5 \%$, Specificity $=73.7 \%)$. The potential for non-invasive sclerotic bone lesion response assessment using non-contrast CT imaging is demonstrated in this study, however, the lack of external validation, retrospective design, and limited patient sample size $(\mathrm{n}=75)$ indicate that further studies are necessary.

\subsubsection{RQS Assessment}

Of the papers identified in this review that used traditional handcrafted features, the mean RQS was $23 \% \pm 19.6 \%$ (range: $0-$ $52.8 \%)$. This low score is the continuation of a trend in radiomics research, where overall low methodological quality has been identified with respect to the RQS by a number of different authors across a variety of radiomics use cases (34-38). Only $17.6 \%$ of studies conducted an external validation of their results (3/17) and only four papers were prospective in nature or had at least a prospective component $(4 / 17,23.5 \%)$. Assessment of feature reproducibility was in general low. Seven studies (7/17, $41.1 \%$ ) performed multiple segmentations to assess robustness of features to each, but only a single paper conducted a phantom study to assess the robustness of features to the scanner variabilities $(1 / 17,5.9 \%)$. Other major limitations identified include: failure to include a calibration statistic and its statistical significance $(15 / 17,88.2 \%)$; the overwhelming lack of accordance to open scientific principles (16/17, 94.1\%); no detection of biological correlates $(0 / 17)$; and no costeffectiveness analysis (0/17). The majority of studies did undertake some form of feature reduction to reduce the chances of overfitting $(12 / 17,70.6 \%)$, and just under half of identified papers included non-radiomics features (such as clinical covariates) into their model building process $(8 / 17$, 47.1\%). Supplementary Table 1 shows how the studies fared with respect to each RQS item.

\subsection{Deep Radiomics}

Utilising deep learning for automated end-to-end salient feature learning, extraction and modelling (which we may term 'Deep Radiomics') instead of traditional hand-crafted features is an area of study that has considerable potential. The rise of the field has been facilitated by an overall increase in the availability of computational resources and toolkits ${ }^{3,4,5}$ that have made the designing and training of these networks easier than ever before. The potential of these models to become crucial pieces of the clinical workflow, supplementing physician decision making and contributing to individualised patient therapy, is enormous. In the deep radiomics publications identified in this review, the detection or classification of patient metastatic lesions is the overwhelming outcome measure.

With respect to imaging modalities, bone scintigraphy was the modality that was most commonly analysed (100, 125-131), and the salient characteristics of these papers are summarised in Table 5. Papandrianos and colleagues (128) designed a CNN architecture for bone metastases diagnosis, where patient bone scintigrams were classified into three classes: no metastasis, degenerative (defined as the absence of metastasis but the presence of degenerative lesions), and metastasis present. Of the 778 patient bone scans examined 15\% were reserved solely for testing and following a thorough exploration of the optimal CNN hyperparameters to be used, the final architecture achieved overall classification accuracy of $91.61 \% \pm 2.46 \%$ (F1-score $=$ 0.938). This result concords with a very similar study undertaken by the same authors, except in this instance only performing a dual-class classification problem (metastasis present vs. metastasis absent) by excluding any patients with degenerative lesions where their $\mathrm{CNN}$ model achieved a higher overall accuracy of $97.38 \%$ (129). Ntakolia et al. (127) performed the same three-class classification problem mentioned above also on $778 \mathrm{PCa}$ patients who underwent bone scintigraphy, except this time deploying a lightweight version of the look-behind FCN (LB-FCN) $(132,133)$ and achieved a better overall accuracy of $97.41 \%$. Their results demonstrated that state-of-the-art classification results can be achieved using a CNN with less learnable parameters and thus requiring less resources for training.

Deep learning techniques can also be utilised to classify individual identified lesions, rather than whole patient images, in bone scintigrams. Cheng et al. (100) used data from 371 breast cancer patients to pre-train a YOLO v4 network (134) to classify identified chest hotspots in bone scintigraphy images as either metastatic or benign. Employing a transfer learning approach, the model was then trained on a dataset of 194 PCa patients under a 10 -fold cross-validation scheme which yielded a lesionlevel classification sensitivity of $0.72 \pm 0.04$ and a precision of $0.9 \pm 0.09$. Their approach suggests the feasibility of utilising metastatic lesions in malignancies other than prostate cancer for pre-training a classification network, thus potentially offering a way for researchers to generate functioning networks even with

\footnotetext{
${ }^{3}$ Keras: https://keras.io/

${ }^{4}$ Tensorflow: https://www.tensorflow.org/

${ }^{5}$ PyTorch: https://pytorch.org/
} 
TABLE 5 | Prominent characteristics of identified mPCa studies using deep radiomics methodology on bone scintigrams.

\begin{tabular}{|c|c|c|c|c|c|c|c|c|}
\hline First Author & Year & Design & $\begin{array}{c}\text { Patient } \\
\text { sample size }\end{array}$ & Patient Cohort & $\begin{array}{c}\text { Deep Network } \\
\text { Architecture }\end{array}$ & Outcome Measures & $\begin{array}{c}\text { External } \\
\text { Validation }\end{array}$ & Results Synopsis \\
\hline Aoki Y (125) & 2020 & $\mathrm{R}$ & 139 & $\mathrm{PCa}$ & Fused dual U-Net & Bone metastases & $\mathrm{N}$ & $\begin{array}{l}\text { Acc }=89.2 \% \\
\text { Sens }=91.7 \% \\
\text { Spec }=87.3 \%\end{array}$ \\
\hline $\begin{array}{l}\text { Cheng D } \\
(100)\end{array}$ & 2021 & $\mathrm{R}$ & 194 & PCa & YOLO v4 & $\begin{array}{l}\text { Chest hotspot } \\
\text { classification }\end{array}$ & $\mathrm{N}$ & $\begin{array}{l}\text { Sens }=0.72 \pm 0.04 \\
\text { Prec }=0.90 \pm 0.04\end{array}$ \\
\hline $\begin{array}{l}\text { Cheng D } \\
(126)\end{array}$ & 2021 & $\mathrm{R}$ & 205 & PCa & $\begin{array}{l}\text { R-CNN \& YOLO } \\
\text { v3 }\end{array}$ & $\begin{array}{l}\text { Chest \& Pelvic hotspot } \\
\text { classification }\end{array}$ & $\mathrm{N}$ & $\begin{array}{l}\text { Chest Classification } \\
\text { Sens }=0.82 \pm 0.08 \\
\text { Prec }=0.70 \pm 0.11 \\
\text { Pelvis Classification } \\
\text { Sens }=0.87 \pm 0.12 \\
\text { Prec }=0.81 \pm 0.11\end{array}$ \\
\hline $\begin{array}{l}\text { Ntakolia C } \\
(127)\end{array}$ & 2020 & $\mathrm{R}$ & 778 & PCa & LB-FCN light & $\begin{array}{l}\text { Bone metastasis } \\
\text { diagnosis }\end{array}$ & $\mathrm{N}$ & $\begin{array}{l}\text { Overall accuracy = } \\
97.41 \%\end{array}$ \\
\hline $\begin{array}{l}\text { Papandrianos } \\
\text { N (128) }\end{array}$ & 2020 & $\mathrm{R}$ & 778 & $\mathrm{PCa}$ & CNN & $\begin{array}{l}\text { Bone Metastasis } \\
\text { Diagnosis }\end{array}$ & $\mathrm{N}$ & $\begin{array}{l}\text { Overall accuracy = } \\
91.61 \% \pm 2.46 \%\end{array}$ \\
\hline $\begin{array}{l}\text { Papandrianos } \\
\text { N (129) }\end{array}$ & 2020 & $\mathrm{R}$ & 586 & $\mathrm{PCa}$ & CNN & $\begin{array}{l}\text { Bone Metastasis } \\
\text { Diagnosis }\end{array}$ & $\mathrm{N}$ & $\begin{array}{l}\text { Overall accuracy = } \\
97.38 \% \\
\text { Sens }=95.8 \%\end{array}$ \\
\hline Sadik M (130) & 2008 & $\mathrm{R}$ & 614 & $\begin{array}{c}\text { PCa patients with suspected } \\
\text { metastatic disease }\end{array}$ & ANN & $\begin{array}{l}\text { Bone Metastasis } \\
\text { Diagnosis }\end{array}$ & $\mathrm{N}$ & $\begin{array}{l}\text { Sens }=90 \% \\
\text { Spec }=89 \%\end{array}$ \\
\hline $\begin{array}{l}\text { Wuestemann } \\
\mathrm{J}(131)\end{array}$ & 2020 & $\mathrm{R}$ & 149 & $\begin{array}{l}\text { PCa patients with malignant } \\
\text { indication on bone scan }\end{array}$ & ANN & $\begin{array}{l}\text { Bone Metastasis } \\
\text { Diagnosis }\end{array}$ & Y & $\begin{array}{l}\text { AUC }=0.937, \text { Sens }= \\
87.0 \% \\
\text { Spec }=98.6 \%, \text { PPV }= \\
98.5 \% \\
\text { NPV }=87.7 \%\end{array}$ \\
\hline
\end{tabular}

Acc, Accuracy; AUC, Area Under Curve; NPV, Negative Predictive Value; P, Prospective; PPV, Positive Predictive Value; R, Retrospective; Sens, Sensitivity; Spec, Specificity.

limited datasets. A follow-up study (126) using instead the YOLO v3 network (135) managed to increase the sensitivity $(0.82 \pm 0.08)$ at the expense of precision $(0.7 \pm 0.11)$ for chest hotspot classification.

The remaining identified studies investigated deep learning on PET and/or CT images with various labelled radiotracers $(99,136-$ 139). Table 6 shows the important characteristics of these studies. Lee et al. examined a cohort of 251 PCa patients with suspected BCR following definitive primary therapy (139). ${ }^{18} \mathrm{~F}$-Fluciclovine PET images were labelled as either 'normal', if no recurrence was found, or 'abnormal', if there was either a recurrence in the prostatic bed or evidence of pelvic metastasis. Two 2D CNN's (ResNet-50) were trained (a slice-based model which predicts individual patient PET slices, and a case-based model that makes a prediction for the entire PET image), and one case-based 3D CNN (ResNet-14) was trained to predict image abnormality. Prediction results on a dedicated test set at the patient level (AUC $=0.75, p=$ 0.013 ; Sens $=85.7 \%$; Spec $=71.4 \%)$ were outperformed by the $2 \mathrm{D}$ slice-based CNN (AUC $=0.971, p<0.001$; Sens $=90.7 \%$; Spec $=$ 95.1\%), however, both 2D models outperformed the 3D ResNet$14(\mathrm{AUC}=0.699, p=0.053$; Sens $=71.4 \%$; Spec $=71.4 \%)$. The authors hypothesise that the reason for the underperformance of the $3 \mathrm{D} C N N$ could be due to the fact that the $3 \mathrm{D} C N N$ had a higher number of learnable parameters and would therefore require a greater training dataset size to generate a sufficiently generalisable model.

One can take this analysis further by classifying individual lesions as benign or malignant. Masoudi et al. (138) demonstrated how deep learning can be used to classify the malignancy of individual bone lesions using only a patient staging CT. An expert radiologist identified, annotated, and segmented 2,880 bone lesions in 114 PCa patients, 41 of which were histopathologically confirmed to be metastatic. Fifteen patients were reserved solely for testing purposes. Using a constructed ensemble model comprised of the 2D ResNet-50 and a 3D ResNet-18 architecture, they achieved high accuracy in classifying bone lesions in the test set $(\mathrm{Acc}=92.2 \%)$. The use of CT to classify the malignancy of patient lesions is not limited exclusively to bone lesions. Hartenstein et al. (137) trained a $\mathrm{CNN}$ for the binary classification of lymph nodes in contrastenhanced CT images of 549 histologically confirmed PCa patients (2,616 labelled lymph nodes identified on ${ }^{68} \mathrm{Ga}$-PSMA PET) and achieved a great accuracy of $89 \%$ (AUC = 0.95; Sens = $86 \%$; Spec $=92 \%)$. External validation of these results was lacking, however, and the authors acknowledge that future studies should utilise histopathology confirmed lymph node invasion via extended pelvic lymph node dissection, rather than ${ }^{68} \mathrm{Ga}$-PSMA PET, as the ground truth reference.

Deep learning can also be applied to perform fully automated detection of metastatic PCa lesions. Automated detection of metastases in patients can relieve some of the significant clinical time burden associated with manual observer analysis of medical images. Zhao et al. (99) conducted a proof-of-concept study for the automated detection and segmentation of metastatic lesions in ${ }^{68} \mathrm{Ga}$-PSMA PET/CT images of mCRPC patients. The authors applied a 2.5D U-Net ensemble network that leveraged information from each anatomical plane separately to make predictions on the presence or absence of metastases in individual voxels, with manual delineations by expert physicians serving as the reference ground truth. Their 
TABLE 6 | Prominent characteristics of identified mPCa papers using deep radiomics methodology on PET and/or CT modalities.

\begin{tabular}{|c|c|c|c|c|c|c|c|c|c|}
\hline $\begin{array}{l}\text { First } \\
\text { Author }\end{array}$ & Year & $\begin{array}{l}\text { Imaging } \\
\text { Series } \\
\text { Analysed }\end{array}$ & Design & $\begin{array}{l}\text { Patient } \\
\text { sample } \\
\text { size }\end{array}$ & Patient Cohort & $\begin{array}{l}\text { Deep Network } \\
\text { Architecture }\end{array}$ & $\begin{array}{l}\text { Outcome } \\
\text { Measures }\end{array}$ & $\begin{array}{c}\text { External } \\
\text { Validation }\end{array}$ & Results Synopsis \\
\hline $\begin{array}{l}\text { Borrelli P } \\
(136)\end{array}$ & 2020 & $\begin{array}{l}{ }^{18} \mathrm{~F}- \\
\text { Choline } \\
\text { PET/CT }\end{array}$ & $\mathrm{R}$ & 399 & Biopsy-proven PCa & $\begin{array}{l}\text { Dual CNN } \\
\text { architecture for } \\
\text { detection \& } \\
\text { segmentation }\end{array}$ & $\begin{array}{l}\text { Lymph node } \\
\text { metastases } \\
\text { detection, } \\
\text { association with } \\
\text { survival }\end{array}$ & $\mathrm{N}$ & $\begin{array}{l}\text { The number of metastatic lymph nodes } \\
\text { detected is associated with PCa- } \\
\text { specific survival ( } \mathrm{HR}=1.19,95 \% \mathrm{Cl} \\
1.05-1.33) \text {. }\end{array}$ \\
\hline Lee J (139) & 2020 & $\begin{array}{l}{ }^{18} \mathrm{~F}- \\
\text { fluciclovine } \\
\text { PET }\end{array}$ & $\mathrm{R}$ & 251 & $\begin{array}{l}\text { PCa patients with } \\
\text { suspected BCR } \\
\text { following definitive } \\
\text { therapy }\end{array}$ & $\begin{array}{l}\text { ResNet-50 \& } \\
\text { ResNet-14 }\end{array}$ & $\begin{array}{l}\text { Pelvic recurrence } \\
\text { or metastasis } \\
\text { classification }\end{array}$ & $\mathrm{N}$ & $\begin{array}{l}\text { Patient-level predictions } \\
\text { AUC }=0.750(p=0.013) \\
\text { Sens }=85.7 \% \\
\text { Spec }=71.4 \% \\
\text { Slice-level predictions } \\
\text { AUC }=0.971(p<0.001) \\
\text { Sens }=90.7 \% \\
\text { Spec }=95.1 \%\end{array}$ \\
\hline $\begin{array}{l}\text { Hartenstein } \\
\text { A (137) }\end{array}$ & 2020 & CT & $\mathrm{R}$ & 549 & $\begin{array}{l}\text { Histologically } \\
\text { confirmed PCa }\end{array}$ & $\mathrm{CNN}$ & LNI & $\mathrm{N}$ & $\begin{array}{l}\text { AUC }=0.95 \\
\text { Sens }=86 \% \\
\text { Acc }=89 \% \\
\text { Spec }=92 \%\end{array}$ \\
\hline $\begin{array}{l}\text { Masoudi S } \\
\text { (138) }\end{array}$ & 2021 & $\mathrm{CT}$ & $\mathrm{R}$ & 114 & $\begin{array}{l}\text { Metastatic PCa } \\
\text { (41 histologically } \\
\text { confirmed) }\end{array}$ & $\begin{array}{l}\text { 2D ResNet-50 \& } \\
\text { 3D ResNet-18 } \\
\text { Ensemble }\end{array}$ & $\begin{array}{l}\text { Bone lesion } \\
\text { classification }\end{array}$ & $\mathrm{N}$ & $\begin{array}{l}\text { Acc }=92.2 \% \\
\text { F1 Score }=92.3 \%\end{array}$ \\
\hline Zhao Y (99) & 2019 & $\begin{array}{l}{ }^{68} \mathrm{Ga}- \\
\mathrm{PSMA} \\
\mathrm{PET} / \mathrm{CT}\end{array}$ & $\mathrm{R}$ & 193 & mCRPC & $\begin{array}{l}\text { 2.5D U-Net } \\
\text { Ensemle }\end{array}$ & $\begin{array}{l}\text { Pelvic area } \\
\text { metastatic lesion } \\
\text { detection }\end{array}$ & $\mathrm{N}$ & $\begin{array}{l}\text { Precision range } 0.79-0.99 \text { depending } \\
\text { on the type of lesion detect (bone, } \\
\text { lymph node, or local) } \\
\text { Sensitivity range } 0.61-0.99\end{array}$ \\
\hline
\end{tabular}

Acc, Accuracy; AUC, Area Under Curve; HR, Hazard Ratio; P, Prospective; R, Retrospective; Sens, Sensitivity; Spec, Specificity.

results, which were restricted only to detecting metastatic disease in the pelvic area, demonstrated great accuracy in the detection of bone lesions (Sens $=0.99$, Prec $=0.99$ ) and lymph node lesions (Sens $=0.9$, Prec $=0.94$ ), but inferior accuracy in the detection of local prostate lesions (Sens $=0.61$, Prec $=0.79$ ). Although highly promising, extending this analysis to detect metastases throughout the body that aren't restricted to the pelvic area remains a fertile pathway of future research. On top of the clear clinical benefit of having lesions detected automatically, there is potentially valuable prognostic information to be gained from the number of automatically detected lesions. Borrelli et al. (136) trained and tested two CNN's, one that detected important patient organs, and another that used this output in conjunction with the patient ${ }^{18} \mathrm{~F}$ Choline PET/CT image to automatically detect lymph node lesions in 399 biopsy-proven PCa patients. They determined that the number of automatically detected lymph node metastases was significantly associated with $\mathrm{PCa}$-specific survival $(\mathrm{HR}=1.19$, $95 \%$ CI 1.05 - 1.33).

\section{DISCUSSION AND FUTURE RECOMMENDATIONS}

\subsection{Quality of the Reviewed Studies}

The RQS assessment of the identified papers reveals some significant limitations in the current landscape of $\mathrm{mPCa}$ radiomics research. The overall lack of prospective methodology and external validation of developed models, along with the other limitations presented above, are crucial contributing factors that are impeding the translation of radiomics models from being of purely academic interest to being properly realised clinical models capable of facilitating truly personalised patient interventions based on the specific phenotype of their malignancy. These downsides should be addressed as soon as possible so that the full potential of radiomics can be realised.

Having said this, it should also be noted that the RQS as a measure of methodological quality has its own limitations. For example, it takes insufficient account of the nature of the study and penalises papers perhaps too harshly in some respects, while not penalising sufficiently other aspects such as significant overfitting. For traditional radiomics papers whose purpose is not to develop a radiomics model, for example, Lin et al. (89) and their investigation into a potential new metric for response assessment, overfitting is not a consideration and thus feature reduction is not necessary. Similarly for studies using machinelearnt features for extraction and modelling, some aspects of the RQS might not be applicable. Employing feature cut-off analyses, for example, is unnecessary for a variety of ML models that are commonly used in radiomics research and, as has been pointed out elsewhere (36), might even compromise the interpretability of the final ML model. Furthermore, once you move to the realm of deep learning for feature extraction, where the black box nature of the algorithm makes the features that are extracted extremely difficult (if not impossible) to interpret, other parts of the RQS criteria become hard to meet. It is difficult to expect researchers to analyse the robustness of individual features to scanner variabilities when the features are deep features that are 
not precisely mathematically defined and very difficult to extract individually from a highly complex deep NN or CNN. Assessing individual feature robustness to segmentation variabilities with deep features is similarly impractical but could also be irrelevant. CNN's, for example, can perform end-to-end feature extraction and predictive modelling on entire medical images without the need for segmentations in the first instance, which should be taken as a significant benefit since it removes what is known to be a large source of bias in radiomics research $(32,53)$. It would perhaps make more sense in these cases to assess robustness at the model level, rather than the individual feature level, but this is not what the RQS criteria specifies.

Considering these factors, we did not think it appropriate to perform RQS assessments on papers that employed deep learning from end-to-end, since it would make for an unfair comparison to those papers that utilised traditional hand-crafted features. However, even without conducting a RQS assessment of the identified deep radiomics studies, several clear methodological weaknesses exist among the reviewed studies. External validation was poor, with only a single study (131) conducting a validation of the model performance on an external test set. None of the papers were prospective in nature. The implicit bias present in retrospective studies, and the near complete lack of external validation of developed models is a large hurdle to the translation of these models into actual clinical practice. Whether using traditional mathematically defined features, or automatically learnt deep features, if radiomics is to achieve its full potential, then these downsides will need to be addressed in future research.

Model validation is a critical point that needs to be underscored. New predictive, prognostic or diagnostic models need to be validated in some fashion and performing this validation exclusively on the same data on which the model was trained will provide an inflated assessment of model performance. Internal validation should be performed as a necessary first step, where cross-validation techniques, bootstrapping, or a hold-out test set from the original study sample are used to assess model performance. Models that perform poorly on an internal validation sample are unlikely to generalise well to previously unseen populations (140). Internal validation alone, however, is insufficient. Radiomics studies should undertake, as a minimum, internal validation, but ideally external validation should also be conducted where possible $(32,33)$. If the purpose is to find robust, informative biomarkers and models that can be utilised in a crossinstitutional fashion for diagnostic or prognostic purposes then it is imperative that external validation is also undertaken. External validation of a developed model or an identified biomarker, in which the predictive performance is assessed on a separate data sample, is necessary to understand their capacity to generalise to data other than that on which they were trained or acquired. The published literature reviewed in the present work demonstrate a poor performance when it comes to external validation, which is directly in line with a myriad of other studies that have highlighted the overall lack of external validation that is present in radiomics research pertaining to other malignancies
$(34,36,37)$. This is an issue that directly impacts the translational capacity of developed models into proper clinical practice, and future studies should seek to address this limitation.

\subsection{Harmonisation and Standardisation for Reproducibility}

The present review has revealed a relative paucity of studies that conducted a feature reproducibility analysis with respect to different segmentation techniques or scanner variabilities. Retrospective or multi-institutional datasets often do not have standardised imaging acquisition and reconstruction protocols. While efforts should be made to obtain a dataset of images with consistent or identical scanner parameters, this is not always feasible. Harmonisation techniques have been employed and validated that limit the influence of heterogeneous imaging parameters on the resulting radiomics signature, which ensures that any variability in the developed model is reflective of the underlying physiology/biology of the imaged lesion instead of being biased by inconsistent scanner protocols. ComBat is a commonly used harmonisation technique that employs an empirical Bayes framework to alter datasets to account for socalled 'batch effects', which refer to confounding experimental factors that affect the output of data other than the underlying biological variations that are of clinical interest (141). The method was originally introduced to reduce the influence of batch effects in genomic microarray research, but its applicability is not limited to this field and has since been utilised in radiomics research. Imaging acquisition protocols and reconstruction methods are batch effects in radiomics research that can be minimised by utilising the ComBat method, and evidence exists supporting its usefulness as a harmonisation technique for imaging modalities such as PET (142), CT $(60,143,144)$, and MRI (145).

While the current evidence points to the usefulness of the ComBat harmonisation method, phantom studies confirming that the method improves feature reproducibility across scanner parameters in specific use cases should be performed where feasible. Indeed, it should be noted that there is no guarantee that the use of ComBat harmonisation, even if the method increases feature reproducibility across variable scanner parameters, will lead to a model with increased diagnostic, prognostic, or predictive power. This is demonstrated in a study by Peeken et al. (116) where the use of ComBat harmonisation resulted in an insignificant AUC value change of 0.01 in logistic regression models used to determine the malignancy status of segmented lymph nodes. Thus, while ComBat harmonisation can have a positive effect on the reproducibility of radiomics features, whether this leads to an improved model will depend on the particular modelling task and should be the subject of future research. Current $\mathrm{mPCa}$ radiomics research has experimented minimally with this technique, and future radiomics studies relating to $\mathrm{mPCa}$ should explore this technique further.

Studies reviewed in the present work utilised a myriad of different radiomics software for feature extraction. This is problematic for reproducibility, since it is known that even when extracting the same imaging biomarker, different 
software can yield different results (146). The standardisation of quantitative imaging biomarker definitions is therefore important to ensure maximum reproducibility. Standardisation initiatives such as the image biomarker standardisation initiative (IBSI) have attempted to address the problem of variable biomarker definitions, producing a set of standardised definitions that researchers can use for their quantitative imaging tasks $(54,147)$. Open source radiomics feature extraction software such as PyRadiomics and RaCaT (148, 149), implemented in the popular coding languages Python and $\mathrm{C}++$, define biomarkers largely in accordance with the IBSI guidelines and carefully document any deviations, which can improve study reproducibility. Not specifying the software used or utilising in-house software that is not provided open source (and is therefore impossible to verify) should be minimised in favour of open-source projects such as those above in future works.

\subsection{Deep Learning - The Future of Radiomics in $\mathrm{mPCa}$ ?}

The reliance on traditional hand-crafted features to characterise ROIs is a limiting factor in radiomics research that can be supplemented by using deep learning methods. Automated feature generation with saliency to the predictive task at hand is a notable benefit that can streamline the radiomics workflow, reducing the need for manual or data-driven feature selection techniques and enable end-to-end predictive modelling with limited human intervention required. The deep radiomics papers reviewed in this work demonstrate the ability of these algorithms to achieve good results in classifying the malignancy of overall patient scans $(127,139)$, classifying individual bone lesions (126), or detecting the anatomical locations of metastatic lesions throughout the patient body (99). As already discussed, external validation is overwhelmingly lacking and needs to be addressed, however, the literature available to date suggest the significant potential for deep learning to contribute positively to the clinical management of mPCa. It should be noted, however, that the overwhelming majority of deep radiomics papers relating to $\mathrm{mPCa}$ developed models with diagnostic applicability. Only a single study attempted to perform any analysis that had prognostic value (136), where they associated the number of automatically detected lymph nodes with PCaspecific survival $(\mathrm{HR}=1.19,95 \% \mathrm{CI} 1.05-1.33)$. The heterogeneity of mPCa and plethora of available treatment options such as hormone therapy, chemotherapy, PSMAlabelled isotope therapy and others make this disease a prime target for deep modelling capable of predicting optimal treatment regimens (150). This remains a relatively unexplored pathway, and future work should certainly delve into this rich area.

Deep learning does not need to be utilised for end-to-end feature extraction and modelling to aid substantively with specific aspects of the radiomics workflow. ROI segmentation is one area where deep learning has already made significant contributions. The recent explosion of fully convolutional networks (FCNs) has led to the development of numerous algorithms capable of performing fully automated segmentation of anatomical structures and patient lesions. FCNs such as the U-Net and its variants have revolutionised the field of medical image segmentation through their ability to output state-of-the-art delineations in seconds (151-153). Kostyszyn et al. (154), for example, have demonstrated the possibility of fully automated prostatic gross tumour volume segmentation on PSMA-PET images in patients with primary PCa. They found good concordance between the fully automated segmentation and the expert manual contour on an external validation cohort, achieving a median dice similarity coefficient (DSC) of 0.81 (range: 0.32-0.95). Other studies have demonstrated the possibility of fully automated and accurate segmentation of the prostate gland and its associated zones with deep learning across various imaging modalities such as CT (155-157) and MRI (158-160). Zhao et al. (99) developed a modified 2.5D U-Net architecture for the automated segmentation of metastatic prostate lesions in ${ }^{68} \mathrm{Ga}$-PSMA PET/CT images in a proof-of-concept study. Their ensemble model, which leveraged information extracted from the axial, sagittal and coronal imaging planes simultaneously, was able to achieve mean DSCs of 0.645 and 0.544 for bone lesions and lymph node lesions when compared to expert manual segmentations, respectively, although the analysis was restricted to metastatic lesions contained in the pelvic area and fully-body validation has yet to be undertaken. By utilising fully automated segmentation algorithms reproducible segmentations can be produced and the issue of inter- and intra-observer variability is resolved, however, further research in this space is necessary because the lack of generalisability of these algorithms to independent datasets remains an impediment to their widespread clinical adoption (87).

Deep learning techniques can yield great benefits, but they are not without their downsides. In all cases the interpretability of the model is compromised relative to the traditional radiomics pathway. While hand-crafted features can often relate to very specific and clinically understandable aspects of tumour biology, deep features are highly abstract and difficult for humans to interpret. A generalised statement on whether this is an acceptable downside cannot be made, as it depends primarily on what the desired outcome of the clinical model is, and whether clinicians value having a greater intuitive understanding of the results of a particular model. Also, the complexity of deep learning models demands greater amounts of training data to produce acceptable results without overfitting. Techniques exist to mitigate against overfitting, such as dropout regularization (161), batch normalization (162) and artificially increasing the size of the dataset through data augmentation. Nevertheless it remains true that the vast majority of current models do not demonstrate generalisability to external datasets, which is evidenced by the studies reviewed in the present work. These downsides need to be considered when future radiomics studies in $\mathrm{mPCa}$ are undertaken.

\subsection{Hybrid Imaging}

There is no inherent limitation on the number of imaging modalities from which radiomics features can be extracted for use in predictive modelling. Hybrid imaging techniques capture 
expanded amounts of information that can be complementary in nature, where each modality characterises different information about the underlying biology of the tumour (163). ${ }^{68} \mathrm{Ga}$-PSMA PET/CT imaging, for example, plays a crucial role in the detection and subsequent clinical management of metastatic PCa (11). ${ }^{68} \mathrm{Ga}$-PSMA PET captures physiological information within the patient's body about the distribution of the PSMA receptor, which is substantially overexpressed in the vast majority of PCa cases (17), while the CT provides highresolution imaging reflecting the underlying density of the patient anatomy. Radiomics features extracted from each of these modalities will thus characterise the heterogeneity of the tumour in different and potentially complementary ways, which could improve model performance. There is evidence that utilising this approach in $\mathrm{mPCa}$ radiomics modelling can yield good results, both in the traditional radiomics methodology (93, 95,98 ) and deep radiomics (99). Particularly in the field of deep radiomics, where the analysis of dual modalities can often be as simple as incorporating an additional channel in the network architecture, this method of analysis should be thoroughly explored.

\section{CONCLUSION}

Radiomics analysis, both using hand-crafted and machinecrafted features has demonstrated significant diagnostic, prognostic, and predictive potential in the clinical management of mPCa. Quality assessment of the identified studies, however,

\section{REFERENCES}

1. Sung H, Ferlay J, Siegel RL, Laversanne M, Soerjomataram I, Jemal A, et al. Global Cancer Statistics 2020: GLOBOCAN Estimates of Incidence and Mortality Worldwide for 36 Cancers in 185 Countries. CA Cancer J Clin (2021) 71(3):209-49. doi: 10.3322/caac.21660

2. Norum J, Nieder C. Treatments for Metastatic Prostate Cancer (mPC): A Review of Costing Evidence. Pharmacoeconomics (2017) 35(12):1223-36. doi: 10.1007/s40273-017-0555-8

3. Chaffer CL, Weinberg RA. A Perspective on Cancer Cell Metastasis. Science (2011) 331(6024):1559-64. doi: 10.1126/science.1203543

4. Svensson E, Christiansen CF, Ulrichsen SP, Rørth MR, Sørensen HT. Survival After Bone Metastasis by Primary Cancer Type: A Danish Population-Based Cohort Study. BMJ Open (2017) 7(9):e016022. doi: 10.1136/bmjopen-2017-016022

5. Daneshmand S, Quek ML, Stein JP, Lieskovsky G, Cai JIE, Pinski J, et al. Prognosis of Patients With Lymph Node Positive Prostate Cancer Following Radical Prostatectomy: Long Term Results. J Urol (2004) 172(6):2252-5. doi: 10.1097/01.ju.0000143448.04161.cc

6. Yadav SS, Stockert JA, Hackert V, Yadav KK, Tewari AK. Intratumor Heterogeneity in Prostate Cancer. Urol Oncol (2018) 36(8):349-60. doi: 10.1016/j.urolonc.2018.05.008

7. Ghafoor S, Burger IA, Vargas AH. Multimodality Imaging of Prostate Cancer. J Nucl Med (2019) 60(10):1350-8. doi: 10.2967/jnumed.119.228320

8. Tourinho-Barbosa RR, Srougi V, Nunes-Silva I, Baghdadi M, Rembeyo G, Eiffel SS, et al. Biochemical Recurrence After Radical Prostatectomy: What Does it Mean? Int Braz J Urol (2018) 44(1):14-21. doi: 10.1590/S16775538.IBJU.2016.0656

9. Mottet N, Bellmunt J, Bolla M, Briers E, Cumberbatch MG, De Santis M, et al. EAU-ESTRO-SIOG Guidelines on Prostate Cancer. Part 1: Screening, Diagnosis, and Local Treatment With Curative Intent. Eur Urol (2016) 71 (4):618-29. doi: 10.1016/j.eururo.2016.08.003 revealed major limitations preventing the implementation of these models in routine clinical practice. Future work should conduct multi-centre and prospective validation of developed radiomics models as a priority to facilitate the clinical translation of radiomics models, so that the full potential of this field can be realised.

\section{AUTHOR CONTRIBUTIONS}

Conception of study: JK, PR, GH, RF, and ME. Collecting literature: JK. Writing Manuscript: JK. Reviewing manuscript: JK, RF, GH, PR, RJ, CK, BR, and ME. All authors contributed to the article and approved the submitted version.

\section{FUNDING}

The authors would like to acknowledge the funding support from the Royal Perth Imaging Research PhD Fellowship (Grant Number 0010121).

\section{SUPPLEMENTARY MATERIAL}

The Supplementary Material for this article can be found online at: https://www.frontiersin.org/articles/10.3389/fonc.2021. 771787/full\#supplementary-material

10. Cui T, Kovell RC, Terlecki RP. Is it Time to Abandon the Digital Recta Examination? Lessons From the PLCO Cancer Screening Trial and PeerReviewed Literature. Curr Med Res Opin (2016) 32(10):1663-9. doi: 10.1080/ 03007995.2016.1198312

11. Lenzo NP, Meyrick D, Turner JH. Review of Gallium-68 PSMA PET/CT Imaging in the Management of Prostate Cancer. Diagnostics (Basel) (2018) 8 (1):16. doi: 10.3390/diagnostics 8010016

12. Thompson IM, Pauler DK, Goodman PJ, Tangen CM, Lucia MS, Parnes HL, et al. Prevalence of Prostate Cancer Among Men With a Prostate-Specific Antigen Level $\leq 4.0$ Ng Per Milliliter. N Engl J Med (2004) 350(22):2239-46. doi: 10.1056/NEJMoa031918

13. Epstein JI, Feng Z, Trock BJ, Pierorazio PM. Upgrading and Downgrading of Prostate Cancer From Biopsy to Radical Prostatectomy: Incidence and Predictive Factors Using the Modified Gleason Grading System and Factoring in Tertiary Grades. Eur Urol (2012) 61(5):1019-24. doi: 10.1016/j.eururo.2012.01.050

14. Van den Wyngaert T, Strobel K, Kampen WU, Kuwert T, van der Bruggen W, Mohan HK, et al. The EANM Practice Guidelines for Bone Scintigraphy. Eur J Nucl Med Mol Imaging (2016) 43(9):1723-38. doi: 10.1007/s00259016-3415-4

15. Pedler K, Kitzing YX, Varol C, Arianayagam M. The Current Status of MRI in Prostate Cancer. Aust Fam Physician (2015) 44(4):225-30.

16. Ahmed HUF, El-Shater Bosaily AM, Brown LCP, Gabe RP, Kaplan RP, Parmar MKP, et al. Diagnostic Accuracy of Multi-Parametric MRI and TRUS Biopsy in Prostate Cancer (PROMIS): A Paired Validating Confirmatory Study. Lancet (2017) 389(10071):815-22. doi: 10.1016/S0140-6736(16)32401-1

17. Rajasekaran SA, Anilkumar G, Oshima E, Bowie JU, Liu H, Heston W, et al. A Novel Cytoplasmic Tail MXXXL Motif Mediates the Internalization of Prostate-Specific Membrane Antigen. Mol Biol Cell (2003) 14(12):4835-45. doi: 10.1091/mbc.E02-11-0731

18. Afshar-Oromieh A, Avtzi E, Giesel FL, Holland-Letz T, Linhart HG, Eder M, et al. The Diagnostic Value of PET/CT Imaging With the 68Ga-Labelled 
PSMA Ligand HBED-CC in the Diagnosis of Recurrent Prostate Cancer. Eur J Nucl Med Mol Imaging (2015) 42(2):197-209. doi: 10.1007/s00259-0142949-6

19. Morigi JJ, Stricker PD, Van Leeuwen PJ, Tang R, Ho B, Nguyen Q, et al. Prospective Comparison of 18F-Fluoromethylcholine Versus 68ga-PSMA PET/CT in Prostate Cancer Patients Who Have Rising PSA After Curative Treatment and are Being Considered for Targeted Therapy. J Nucl Med (2015) 56(8):1185-90. doi: 10.2967/jnumed.115.160382

20. Kumar V, Gu Y, Basu S, Berglund A, Eschrich SA, Schabath MB, et al. Radiomics: The Process and the Challenges. Magn Reson Imaging (2012) 30 (9):1234-48. doi: 10.1016/j.mri.2012.06.010

21. Gillies RJ, Kinahan PE, Hricak H. Radiomics: Images Are More Than Pictures, They Are Data. Radiology (2016) 278(2):563-77. doi: 10.1148/ radiol.2015151169

22. Yip SSF, Aerts HJWL. Applications and Limitations of Radiomics. Phys Med Biol (2016) 61(13):R150-R66. doi: 10.1088/0031-9155/61/13/R150

23. Zhao B, Lee SM, Lee H-J, Tan Y, Qi J, Persigehl T, et al. Variability in Assessing Treatment Response: Metastatic Colorectal Cancer as a Paradigm. Clin Cancer Res (2014) 20(13):3560-8. doi: 10.1158/10780432.CCR-14-0245

24. Mazor RD, Savir A, Gheorghiu D, Weinstein Y, Abadi-Korek I, Shabshin N. The Inter-Observer Variability of Breast Density Scoring Between Mammography Technologists and Breast Radiologists and its Effect on the Rate of Adjuvant Ultrasound. Eur J Radiol (2016) 85(5):957-62. doi: 10.1016/j.ejrad.2016.02.023

25. Djavan BOB, Waldert M, Zlotta A, Dobronski P, Seitz C, Remzi M, et al. Safety and Morbidity of First and Repeat Transrectal Ultrasound Guided Prostate Needle Biopsies: Results of a Prospective European Prostate Cancer Detection Study. J Urol (2001) 166(3):856-60. doi: 10.1016/S0022-5347(05) 65851-X

26. Lambin P, Rios-Velazquez E, Leijenaar R, Carvalho S, van Stiphout RGPM, Granton P, et al. Radiomics: Extracting More Information From Medical Images Using Advanced Feature Analysis. Eur J Cancer (1990) (2011) 48 (4):441-6. doi: 10.1016/j.ejca.2011.11.036

27. Asselin M-C, O'Connor JPB, Boellaard R, Thacker NA, Jackson A. Quantifying Heterogeneity in Human Tumours Using MRI and PET. Eur J Cancer (2012) 48(4):447-55. doi: 10.1016/j.ejca.2011.12.025

28. Sun Y, Reynolds HM, Parameswaran B, Wraith D, Finnegan ME, Williams S, et al. Multiparametric MRI and Radiomics in Prostate Cancer: A Review. Australas Phys Eng Sci Med (2019) 42(1):3-25. doi: 10.1007/s13246-01900730-z

29. Xue C, Zhou Y, Lo GG, Wong OL, Yu SK, Cheung KY, et al. Reliability of Radiomics Features Due to Image Reconstruction Using a Standardized T2Weighted Pulse Sequence for MR-Guided Radiotherapy: An Anthropomorphic Phantom Study. Magn Reson Med (2021) 85(6):343446. doi: $10.1002 / \mathrm{mrm} .28650$

30. Shafiq-Ul-Hassan M, Latifi K, Zhang G, Ullah G, Gillies R, Moros E. Voxel Size and Gray Level Normalization of CT Radiomic Features in Lung Cancer. Sci Rep (2018) 8(1):10545-9. doi: 10.1038/s41598-018-28895-9

31. Zhao B. Understanding Sources of Variation to Improve the Reproducibility of Radiomics. Front Oncol (2021) 11:633176. doi: 10.3389/fonc.2021.633176

32. Lambin P, Leijenaar RTH, Deist TM, Peerlings J, de Jong EEC, van Timmeren J, et al. Radiomics: The Bridge Between Medical Imaging and Personalized Medicine. Nat Rev Clin Oncol (2017) 14(12):749-62. doi: 10.1038/nrclinonc.2017.141

33. Collins GS, Reitsma JB, Altman DG, Moons KGM. Transparent Reporting of a Multivariable Prediction Model for Individual Prognosis or Diagnosis (TRIPOD) : The TRIPOD Statement. BMJ (2015) 350:g7594. doi: 10.1136/ bmj.g7594

34. Park JE, Kim D, Kim HS, Park SY, Kim JY, Cho SJ, et al. Quality of Science and Reporting of Radiomics in Oncologic Studies: Room for Improvement According to Radiomics Quality Score and TRIPOD Statement. Eur Radiol (2020) 30(1):523-36. doi: 10.1007/s00330-019-06360-z

35. Fiz F, Viganò L, Gennaro N, Costa G, La Bella L, Boichuk A, et al. Radiomics of Liver Metastases: A Systematic Review. Cancers (Basel) (2020) 12(10):124. doi: $10.3390 /$ cancers 12102881

36. Stanzione A, Gambardella M, Cuocolo R, Ponsiglione A, Romeo V, Imbriaco M. Prostate MRI Radiomics: A Systematic Review and Radiomic Quality
Score Assessment. Eur J Radiol (2020) 129:109095. doi: 10.1016/ j.ejrad.2020.109095

37. Park JE, Kim HS, Kim D, Park SY, Kim JY, Cho SJ, et al. A Systematic Review Reporting Quality of Radiomics Research in Neuro-Oncology: Toward Clinical Utility and Quality Improvement Using High-Dimensional Imaging Features. BMC Cancer (2020) 20(1):29. doi: 10.1186/s12885-0196504-5

38. Chetan MR, Gleeson FV. Radiomics in Predicting Treatment Response in non-Small-Cell Lung Cancer: Current Status, Challenges and Future Perspectives. Eur Radiol (2021) 31(2):1049-58. doi: 10.1007/s00330-02007141-9

39. Zhao B, Tan Y, Tsai W-Y, Qi J, Xie C, Lu L, et al. Reproducibility of Radiomics for Deciphering Tumor Phenotype With Imaging. Sci Rep (2016) 6(1):23428. doi: 10.1038/srep23428

40. Shafiq-ul-Hassan M, Zhang GG, Latifi K, Ullah G, Hunt DC, Balagurunathan Y, et al. Intrinsic Dependencies of CT Radiomic Features on Voxel Size and Number of Gray Levels. Med Phys (2017) 44(3):1050-62. doi: $10.1002 / \mathrm{mp} .12123$

41. Zwanenburg A. Radiomics in Nuclear Medicine: Robustness, Reproducibility, Standardization, and How to Avoid Data Analysis Traps and Replication Crisis. Eur J Nucl Med Mol Imaging (2019) 46(13):2638-55. doi: 10.1007/s00259-019-04391-8

42. Midya A, Chakraborty J, Gönen M, Do RKG, Simpson AL. Influence of CT Acquisition and Reconstruction Parameters on Radiomic Feature Reproducibility. J Med Imaging (2018) 5(1):11020. doi: 10.1117/ 1.JMI.5.1.011020

43. Berenguer R, Del Rosario Pastor-Juan M, Canales-Vázquez J, Castro-García M, Villas MV, Legorburo FM, et al. Radiomics of CT Features may be Nonreproducible and Redundant: Influence of CT Acquisition Parameters. Radiology (2018) 288(2):407-15. doi: 10.1148/radiol.2018172361

44. Li X, Chen E, Guo B, Yang W, Han R, Hu C, et al. The Impact of Respiratory Motion and CT Pitch on the Robustness of Radiomics Feature Extraction in 4DCT Lung Imaging. Comput Methods Programs BioMed (2020) 197:105719. doi: 10.1016/j.cmpb.2020.105719

45. Larue RTHM, van Timmeren JE, de Jong EEC, Feliciani G, Leijenaar RTH, Schreurs WMJ, et al. Influence of Gray Level Discretization on Radiomic Feature Stability for Different CT Scanners, Tube Currents and Slice Thicknesses: A Comprehensive Phantom Study. Acta Oncol (2017) 56 (11):1544-53. doi: 10.1080/0284186X.2017.1351624

46. Mahmood U, Apte A, Deasy JO, Schmidtlein CR, Shukla-Dave A. Investigating the Robustness Neighborhood Grey Tone Difference Matrix (NGTDM) and Grey Level Co-Occurrence Matrix (GLCM) Radiomic Features on Clinical Computed Tomography Systems Using Anthropomorphic Phantoms: Evidence From a Multivendor Study. J Comput Assist Tomogr (2017) 41(6):995. doi: 10.1097/RCT.0000000000000632

47. Park JE, Park SY, Kim HJ, Kim HS. Reproducibility and Generalizability in Radiomics Modeling: Possible Strategies in Radiologic and Statistical Perspectives. Korean J Radiol (2019) 20(7):1124-37. doi: 10.3348/ kjr.2018.0070

48. Shur J, Blackledge M, D’Arcy J, Collins DJ, Bali M, O’Leach M, et al. MRI Texture Feature Repeatability and Image Acquisition Factor Robustness, a Phantom Study and in Silico Study. Eur Radiol Exp (2021) 5(1):2. doi: 10.1186/s41747-020-00199-6

49. Yang F, Dogan N, Stoyanova R, Ford JC. Evaluation of Radiomic Texture Feature Error Due to MRI Acquisition and Reconstruction: A Simulation Study Utilizing Ground Truth. Phys Med (2018) 50:26-36. doi: 10.1016/ j.ejmp.2018.05.017

50. Molina D, Pérez-Beteta J, Martínez-González A, Martino J, Velasquez C, Arana E, et al. Influence of Gray Level and Space Discretization on Brain Tumor Heterogeneity Measures Obtained From Magnetic Resonance Images. Comput Biol Med (2016) 78:49-57. doi: 10.1016/j.compbiomed. 2016.09.011

51. Montagne S, Hamzaoui D, Allera A, Ezziane M, Luzurier A, Quint R, et al. Challenge of Prostate MRI Segmentation on T2-Weighted Images: InterObserver Variability and Impact of Prostate Morphology. Insights Imaging (2021) 12(1):71. doi: 10.1186/s13244-021-01010-9

52. Fiorino C, Reni M, Bolognesi A, Cattaneo GM, Calandrino R. Intra- and Inter-Observer Variability in Contouring Prostate and Seminal Vesicles: 
Implications for Conformal Treatment Planning. Radiother Oncol (1998) 47 (3):285-92. doi: 10.1016/S0167-8140(98)00021-8

53. Balagurunathan Y, Gu Y, Wang H, Kumar V, Grove O, Hawkins S, et al. Reproducibility and Prognosis of Quantitative Features Extracted From CT Images. Transl Oncol (2014) 7(1):72-87. doi: 10.1593/tlo.13844

54. Zwanenburg A, Leger S, Vallières M, Löck S. Image Biomarker Standardisation Initiative. arXiv:161207003 (2016).

55. Leijenaar RTH, Nalbantov G, Carvalho S, van Elmpt WJC, Troost EGC, Boellaard R, et al. The Effect of SUV Discretization in Quantitative FDGPET Radiomics: The Need for Standardized Methodology in Tumor Texture Analysis. Sci Rep (2015) 5(1):11075. doi: 10.1038/srep11075

56. Altazi BA, Zhang GG, Fernandez DC, Montejo ME, Hunt D, Werner J, et al. Reproducibility of F18-FDG PET Radiomic Features for Different Cervical Tumor Segmentation Methods, Gray-Level Discretization, and Reconstruction Algorithms. J Appl Clin Med Phys (2017) 18(6):32-48. doi: $10.1002 / \mathrm{acm} 2.12170$

57. Hatt M, Tixier F, Pierce L, Kinahan PE, Le Rest CC, Visvikis D. Characterization of PET/CT Images Using Texture Analysis: The Past, the Present... Any Future? Eur J Nucl Med Mol Imaging (2017) 44(1):151-65. doi: 10.1007/s00259-016-3427-0

58. van Velden FHP, Kramer GM, Frings V, Nissen IA, Mulder ER, de Langen AJ, et al. Repeatability of Radiomic Features in Non-Small-Cell Lung Cancer [F-18]FDG-PET/CT Studies: Impact of Reconstruction and Delineation. Mol Imaging Biol (2016) 18(5):788-95. doi: 10.1007/s11307-016-0940-2

59. Duron L, Balvay D, Perre SV, Bouchouicha A, Savatovsky J, Sadik J-C, et al. Gray-Level Discretization Impacts Reproducible MRI Radiomics Texture Features. PloS One (2019) 14(3):e0213459. doi: 10.1371/journal.pone. 0213459

60. Ibrahim A, Refaee T, Primakov S, Barufaldi B, Acciavatti RJ, Granzier RWY, et al. The Effects of In-Plane Spatial Resolution on CT-Based Radiomic Features' Stability With and Without ComBat Harmonization. Cancers (Basel) (2021) 13(8):1848. doi: 10.3390/cancers13081848

61. Whybra P, Parkinson C, Foley K, Staffurth J, Spezi E. Assessing Radiomic Feature Robustness to Interpolation in 18F-FDG PET Imaging. Sci Rep (2019) 9(1):1-10. doi: 10.1038/s41598-019-46030-0

62. Park S-H, Lim H, Bae BK, Hahm MH, Chong GO, Jeong SY, et al. Robustness of Magnetic Resonance Radiomic Features to Pixel Size Resampling and Interpolation in Patients With Cervical Cancer. Cancer Imaging (2021) 21(1):19. doi: 10.1186/s40644-021-00388-5

63. Avanzo M, Wei L, Stancanello J, Vallieres M, Rao A, Morin O, et al. Machine and Deep Learning Methods for Radiomics. Med Phys (2020) 47(5):e185202. doi: $10.1002 / \mathrm{mp} .13678$

64. LeCun Y, Bengio Y, Hinton G. Deep Learning. Nature (2015) 521 (7553):436-44. doi: 10.1038/nature14539

65. Naqa IE. The Role of Quantitative PET in Predicting Cancer Treatment Outcomes. Clin Transl Imaging (2014) 2(4):305-20. doi: 10.1007/s40336014-0063-1

66. Wei L, El Naqa I. Feature Extraction and Qualification. In: Radiomics and Radiogenomics. Boca Raton: Chapman and Hall/CRC (2019). p. 121-49.

67. Jain AK. Fundamentals of Digital Image Processing. Englewood Cliffs: Prentice-Hall (1989).

68. Haralick RM, Shanmugam K, Dinstein IH. Textural Features for Image Classification. IEEE Trans Syst Man Cybern (1973) 6):610-21. doi: 10.1109/ TSMC.1973.4309314

69. Galloway MM. Texture Analysis Using Gray Level Run Lengths. Comput Gr Image Process (1975) 4(2):172-9. doi: 10.1016/S0146-664X(75)80008-6

70. Thibault G, Angulo J, Meyer F. Advanced Statistical Matrices for Texture Characterization: Application to Cell Classification. IEEE Trans BioMed Eng (2014) 61(3):630-7. doi: 10.1109/TBME.2013.2284600

71. Amadasun M, King R. Textural Features Corresponding to Textural Properties. IEEE Trans Syst Man Cybern (1989) 19(5):1264-74. doi: $10.1109 / 21.44046$

72. Sun C, Wee WG. Neighboring Gray Level Dependence Matrix for Texture Classification. Comput Gr Image Process (1983) 23(3):341-52. doi: 10.1016/ 0734-189X(83)90032-4

73. Rizzo S, Botta F, Raimondi S, Origgi D, Fanciullo C, Morganti AG, et al. Radiomics: The Facts and the Challenges of Image Analysis. Eur Radiol Exp (2018) 2(1):1-8. doi: 10.1186/s41747-018-0068-z
74. Fogel I, Sagi D. Gabor Filters as Texture Discriminator. Biol Cybern (1989) 61(2):103-13. doi: 10.1007/BF00204594

75. Addison PS. The Illustrated Wavelet Transform Handbook. In: B Raton, editor. Introductory Theory and Applications in Science, Engineering, Medicine and Finance, 2nd ed. Boca Raton: Taylor \& Francis Group (2016).

76. Noortman WA, Vriens D, Slump CH, Bussink J, Meijer TW, de Geus-Oei LF, et al. Adding the Temporal Domain to PET Radiomic Features. PloS One (2020) 15(9):e0239438. doi: 10.1371/journal.pone.0239438

77. Goodfellow I, Bengio Y, Courville A. Deep Learning. Cambridge: MIT press (2016).

78. MD Zeiler and R Fergus eds. Visualizing and Understanding Convolutional Networks. In: European Conference on Computer Vision. Zurich: Springer.

79. Shang S, Sun J, Yue Z, Wang Y, Wang X, Luo Y, et al. Multi-Parametric MRI Based Radiomics With Tumor Subregion Partitioning for Differentiating Benign and Malignant Soft-Tissue Tumors. BioMed Signal Process Control (2021) 67:102522. doi: 10.1016/j.bspc.2021.102522

80. Lao J, Chen Y, Li Z-C, Li Q, Zhang J, Liu J, et al. A Deep Learning-Based Radiomics Model for Prediction of Survival in Glioblastoma Multiforme. Sci Rep (2017) 7(1):10353-8. doi: 10.1038/s41598-017-10649-8

81. Avanzo M, Stancanello J, Pirrone G, Sartor G. Radiomics and Deep Learning in Lung Cancer. Strahlenther Onkol (2020) 196(10):879-87. doi: 10.1007/ s00066-020-01625-9

82. Hou Y, Bao J, Song Y, Bao M-L, Jiang K-W, Zhang J, et al. Integration of Clinicopathologic Identification and Deep Transferrable Image Feature Representation Improves Predictions of Lymph Node Metastasis in Prostate Cancer. EBioMedicine (2021) 68:103395. doi: 10.1016/ j.ebiom.2021.103395

83. Stevens LM, Mortazavi BJ, Deo RC, Curtis L, Kao DP. Recommendations for Reporting Machine Learning Analyses in Clinical Research. Circ Cardiovasc Qual Outcomes (2020) 13(10):e006556. doi: 10.1161/CIRCOUTCOMES. 120.006556

84. Yao S, Jiang H, Song B. Radiomics in Prostate Cancer: Basic Concepts and Current State-of-the-Art. Chin J Acad Radiol (2020) 2(3):47-55. doi: 10.1007/s42058-019-00020-3

85. Sullivan DC, Obuchowski NA, Kessler LG, Raunig DL, Gatsonis C, Huang EP, et al. Metrology Standards for Quantitative Imaging Biomarkers. Radiology (2015) 277(3):813-25. doi: 10.1148/radiol.2015142202

86. Osman SOS, Leijenaar RTH, Cole AJ, Lyons CA, Hounsell AR, Prise KM, et al. Computed Tomography-Based Radiomics for Risk Stratification in Prostate Cancer. Int J Radiat Oncol Biol Phys (2019) 105(2):448-56. doi: 10.1016/j.ijrobp.2019.06.2504

87. van Timmeren JE, Cester D, Tanadini-Lang S, Alkadhi H, Baessler B. Radiomics in Medical Imaging-"How-to" Guide and Critical Reflection. Insights Imaging (2020) 11(1):91. doi: 10.1186/s13244-020-00887-2

88. Obuchowski NA, Reeves AP, Huang EP, Wang X-F, Buckler AJ, Kim HJ, et al. Quantitative Imaging Biomarkers: A Review of Statistical Methods for Computer Algorithm Comparisons. Stat Methods Med Res (2015) 24(1):68106. doi: $10.1177 / 0962280214537390$

89. Lin C, Harmon S, Bradshaw T, Eickhoff J, Perlman S, Liu G, et al. ResponseTo-Repeatability of Quantitative Imaging Features for Longitudinal Response Assessment. Phys Med Biol (2019) 64(2):025019. doi: 10.1088/ 1361-6560/aafa0a

90. Tzeng S, Zhu J, Weisman AJ, Bradshaw TJ, Jeraj R. Spatial Process Decomposition for Quantitative Imaging Biomarkers Using Multiple Images of Varying Shapes. Stat Med (2021) 40(5):1243-61. doi: 10.1002/ sim. 8838

91. Liu X, Khalvati F, Namdar K, Fischer S, Lewis S, Taouli B, et al. Can Machine Learning Radiomics Provide Pre-Operative Differentiation of Combined Hepatocellular Cholangiocarcinoma From Hepatocellular Carcinoma and Cholangiocarcinoma to Inform Optimal Treatment Planning? Eur Radiol (2021) 31(1):244-55. doi: 10.1007/s00330-020-07119-7

92. Zhang Y, Oikonomou A, Wong A, Haider MA, Khalvati F. Radiomics-Based Prognosis Analysis for non-Small Cell Lung Cancer. Sci Rep (2017) 7(1):1-8 doi: $10.1038 /$ srep46349

93. Damascelli A, Gallivanone F, Cristel G, Cava C, Interlenghi M, Esposito A, et al. Advanced Imaging Analysis in Prostate MRI: Building a Radiomic Signature to Predict Tumor Aggressiveness. Diagnostics (2021) 11(4):594. doi: 10.3390/diagnostics11040594 
94. Wang Y, Yu B, Zhong F, Guo Q, Li K, Hou Y, et al. MRI-Based Texture Analysis of the Primary Tumor for Pre-Treatment Prediction of Bone Metastases in Prostate Cancer. Magn Reson Imaging (2019) 60:76-84. doi: 10.1016/j.mri.2019.03.007

95. Zhang W, Mao N, Wang Y, Xie H, Duan S, Zhang X, et al. A Radiomics Nomogram for Predicting Bone Metastasis in Newly Diagnosed Prostate Cancer Patients. Eur J Radiol (2020) 128:109020. doi: 10.1016/ j.ejrad.2020.109020

96. Acar E, Leblebici A, Ellidokuz BE, Basbinar Y, Kaya GC. Machine Learning for Differentiating Metastatic and Completely Responded Sclerotic Bone Lesion in Prostate Cancer: A Retrospective Radiomics Study. Br J Radiol (2019) 92(1101):20190286. doi: 10.1259/bjr.20190286

97. Cysouw MCF, Jansen BHE, van de Brug T, Oprea-Lager DE, Pfaehler E, de Vries BM, et al. Machine Learning-Based Analysis of [18F]DCFPyL PET Radiomics for Risk Stratification in Primary Prostate Cancer. Eur J Nucl Med Mol Imaging (2021) 48(2):340-9. doi: 10.1007/s00259-020-04971-z

98. Perk T, Bradshaw T, Chen S, Im H-J, Cho S, Perlman S, et al. Automated Classification of Benign and Malignant Lesions in 18 F-NaF PET/CT Images Using Machine Learning. Phys Med Biol (2018) 63(22):225019. doi: 10.1088/ 1361-6560/aaebd0

99. Zhao Y, Gafita A, Vollnberg B, Tetteh G, Haupt F, Afshar-Oromieh A, et al. Deep Neural Network for Automatic Characterization of Lesions on 68GaPSMA-11 PET/CT. Eur J Nucl Med Mol Imaging (2020) 47(3):603-13. doi: 10.1007/s00259-019-04606-y

100. Cheng D-C, Hsieh T-C, Yen K-Y, Kao C-H. Lesion-Based Bone Metastasis Detection in Chest Bone Scintigraphy Images of Prostate Cancer Patients Using Pre-Train, Negative Mining, and Deep Learning. Diagnostics (2021) 11(3):518. doi: 10.3390/diagnostics11030518

101. Horwich A, Parker C, de Reijke T, Kataja V. Prostate Cancer: ESMO Clinical Practice Guidelines for Diagnosis, Treatment and Follow-Up. Ann Oncol (2013) 24(Suppl. 6):106-14. doi: 10.1093/annonc/mdt208

102. Vickers AJ, Ulmert D, Sjoberg DD, Bennette CJ, Björk T, Gerdtsson A, et al. Strategy for Detection of Prostate Cancer Based on Relation Between Prostate Specific Antigen at Age 40-55 and Long Term Risk of Metastasis: Case-Control Study. BMJ (2013) 346(7907):27-f2023. doi: 10.1136/bmj.f2023

103. Khurshid Z, Ahmadzadehfar H, Gaertner FC, Papp L, Zsóter N, Essler M, et al. Role of Textural Heterogeneity Parameters in Patient Selection for 177Lu-PSMA Therapy. via response prediction Oncotarget (2018) 9 (70):33312-21. doi: 10.18632/oncotarget.26051

104. Rusthoven CGMD, Carlson JAMD, Waxweiler TVMD, Yeh NMDPD, Raben DMD, Flaig TWMD, et al. The Prognostic Significance of Gleason Scores in Metastatic Prostate Cancer. Urol Oncol (2014) 32(5):707-13. doi: 10.1016/j.urolonc.2014.01.004

105. Loblaw DA, Virgo KS, Nam R, Somerfield MR, Ben-Josef E, Mendelson DS, et al. Initial Hormonal Management of Androgen-Sensitive Metastatic, Recurrent, or Progressive Prostate Cancer: 2006 Update of an American Society of Clinical Oncology Practice Guideline. J Clin Oncol (2007) 25 (12):1596-605. doi: 10.1200/JCO.2006.10.1949

106. Halabi S, Small EJ, Kantoff PW, Kattan MW, Kaplan EB, Dawson NA, et al. Prognostic Model for Predicting Survival in Men With Hormone-Refractory Metastatic Prostate Cancer. J Clin Oncol (2003) 21(7):1232-7. doi: 10.1200/ JCO.2003.06.100

107. Armstrong AJ, Halabi S, Luo J, Nanus DM, Giannakakou P, Szmulewitz RZ, et al. Prospective Multicenter Validation of Androgen Receptor Splice Variant 7 and Hormone Therapy Resistance in High-Risk CastrationResistant Prostate Cancer: The PROPHECY Study. J Clin Oncol (2019) 37 (13):1120-9. doi: 10.1200/JCO.18.01731

108. Oyama N, Akino H, Suzuki Y, Kanamaru H, Miwa Y, Tsuka H, et al. Prognostic Value of 2-Deoxy-2-[F-18] Fluoro-D-Glucose Positron Emission Tomography Imaging for Patients With Prostate Cancer. Mol Imaging Biol (2002) 4(1):99-104. doi: 10.1016/s1095-0397(01)00065-6

109. Meirelles GSP, SchÖDer H, Ravizzini GC, GÖNen M, Fox JJ, Humm J, et al. Prognostic Value of Baseline [18f] Fluorodeoxyglucose Positron Emission Tomography and 99mtc-MDP Bone Scan in Progressing Metastatic Prostate Cancer. Clin Cancer Res (2010) 16(24):6093-9. doi: 10.1158/10780432.CCR-10-1357

110. Bauckneht M, Bertagna F, Donegani MI, Durmo R, Miceli A, De Biasi V, et al. The Prognostic Power of 18F-FDG PET/CT Extends to Estimating
Systemic Treatment Response Duration in Metastatic Castration-Resistant Prostate Cancer (mCRPC) Patients. Prostate Cancer Prostatic Dis (2021). doi: 10.1038/s41391-021-00391-8

111. Moher D, Liberati A, Tetzlaff J, Altman DG. Preferred Reporting Items for Systematic Reviews and Meta-Analyses: The PRISMA Statement. BMJ (2009) 339(7716):e78-336. doi: 10.1136/bmj.b2535

112. Alongi P, Stefano A, Comelli A, Laudicella R, Scalisi S, Arnone G, et al. Radiomics Analysis of 18F-Choline PET/CT in the Prediction of Disease Outcome in High-Risk Prostate Cancer: An Explorative Study on Machine Learning Feature Classification in 94 Patients. Eur Radiol (2021) 31(7):4595605. doi: 10.1007/s00330-020-07617-8

113. Hayakawa T, Tabata K-i, Tsumura H, Kawakami S, Katakura T, Hashimoto $\mathrm{M}$, et al. Size of Pelvic Bone Metastasis as a Significant Prognostic Factor for Metastatic Prostate Cancer Patients. Japanese J Radiol (2020) 38(10):993-6. doi: 10.1007/s11604-020-01004-5

114. Moazemi S, Khurshid Z, Erle A, Lütje S, Essler M, Schultz T, et al. Machine Learning Facilitates Hotspot Classification in PSMA-PET/CT With Nuclear Medicine Specialist Accuracy. Diagnostics (2020) 10(9):622. doi: 10.3390/ diagnostics10090622

115. Moazemi S, Erle A, Lütje S, Gaertner FC, Essler M, Bundschuh RA. Estimating the Potential of Radiomics Features and Radiomics Signature From Pretherapeutic PSMA-PET-CT Scans and Clinical Data for Prediction of Overall Survival When Treated With 177Lu-PSMA. Diagnostics (2021) 11 (2):186. doi: 10.3390/diagnostics 11020186

116. Peeken JC, Shouman MA, Kroenke M, Rauscher I, Maurer T, Gschwend JE, et al. A CT-Based Radiomics Model to Detect Prostate Cancer Lymph Node Metastases in PSMA Radioguided Surgery Patients. Eur J Nucl Med Mol Imaging (2020) 47(13):2968-77. doi: 10.1007/s00259-020-04864-1

117. Zamboglou C, Carles M, Fechter T, Kiefer S, Reichel K, Fassbender TF, et al. Radiomic Features From PSMA PET for non-Invasive Intraprostatic Tumor Discrimination and Characterization in Patients With Intermediate- and High-Risk Prostate Cancer - A Comparison Study With Histology Reference. Theranostics (2019) 9(9):2595-605. doi: 10.7150/thno.32376

118. Li L, Shiradkar R, Leo P, Algohary A, Fu P, Tirumani SH, et al. A Novel Imaging Based Nomogram for Predicting Post-Surgical Biochemical Recurrence and Adverse Pathology of Prostate Cancer From Pre-Operative Bi-Parametric MRI. EBioMedicine (2021) 63:103163. doi: 10.1016/ j.ebiom.2020.103163

119. Reischauer C, Patzwahl R, Koh D-M, Froehlich JM, Gutzeit A. Texture Analysis of Apparent Diffusion Coefficient Maps for Treatment Response Assessment in Prostate Cancer Bone Metastases-A Pilot Study. Eur J Radiol (2018) 101:184-90. doi: 10.1016/j.ejrad.2018.02.024

120. Musch M, Klevecka V, Roggenbuck U, Kroepfl D. Complications of Pelvic Lymphadenectomy in 1,380 Patients Undergoing Radical Retropubic Prostatectomy Between 1993 and 2006. J Urol (2008) 179(3):923-9. doi: 10.1016/j.juro.2007.10.072

121. Fedorov A, Schwier M, Clunie D, Herz C, Pieper S, Kikinis R, et al. An Annotated Test-Retest Collection of Prostate Multiparametric MRI. Sci Data (2018) 5(1):180281-13. doi: 10.1038/sdata.2018.281

122. Briganti A, Larcher A, Abdollah F, Capitanio U, Gallina A, Suardi N, et al. Updated Nomogram Predicting Lymph Node Invasion in Patients With Prostate Cancer Undergoing Extended Pelvic Lymph Node Dissection: The Essential Importance of Percentage of Positive Cores. Eur Urol (2011) 61 (3):480-7. doi: 10.1016/j.eururo.2011.10.044

123. Perk T, Chen S, Harmon S, Lin C, Bradshaw T, Perlman S, et al. A Statistically Optimized Regional Thresholding Method (SORT) for Bone Lesion Detection in F-18-NaF PET/CT Imaging. Phys Med Biol (2018) 63 (22):225018. doi: 10.1088/1361-6560/aaebba

124. Ferdinandus J, Eppard E, Gaertner FC, Kürpig S, Fimmers R, Yordanova A, et al. Predictors of Response to Radioligand Therapy of Metastatic CastrateResistant Prostate Cancer With 177Lu-PSMA-617. J Nucl Med (2017) 58 (2):312-9. doi: 10.2967/jnumed.116.178228

125. Aoki Y, Nakayama M, Nomura K, Tomita Y, Nakajima K, Yamashina M, et al. The Utility of a Deep Learning-Based Algorithm for Bone Scintigraphy in Patient With Prostate Cancer. Ann Nucl Med (2020) 34(12):926-31. doi: 10.1007/s12149-020-01524-0

126. Cheng D-C, Liu C-C, Hsieh T-C, Yen K-Y, Kao C-H. Bone Metastasis Detection in the Chest and Pelvis From a Whole-Body Bone Scan Using 
Deep Learning and a Small Dataset. Electronics (2021) 10(10):1201. doi: 10.3390/electronics10101201

127. Ntakolia C, Diamantis DE, Papandrianos N, Moustakidis S, Papageorgiou EI. A Lightweight Convolutional Neural Network Architecture Applied for Bone Metastasis Classification in Nuclear Medicine: A Case Study on Prostate Cancer Patients. Healthcare (2020) 8(4):493. doi: 10.3390/healthcare8040493

128. Papandrianos N, Papageorgiou E, Anagnostis A, Papageorgiou K. Efficient Bone Metastasis Diagnosis in Bone Scintigraphy Using a Fast Convolutional Neural Network Architecture. Diagnostics (2020) 10(8):532. doi: 10.3390/ diagnostics10080532

129. Papandrianos N, Papageorgiou E, Anagnostis A, Papageorgiou K. Bone Metastasis Classification Using Whole Body Images From Prostate Cancer Patients Based on Convolutional Neural Networks Application. PloS One (2020) 15(8):e0237213. doi: 10.1371/journal.pone.0237213

130. Sadik M, Hamadeh I, Nordblom P, Suurkula M, Hoglund P, Ohlsson M, et al. Computer-Assisted Interpretation of Planar Whole-Body Bone Scans. J Nucl Med (2008) 49(12):1958-65. doi: 10.2967/jnumed.108.055061

131. Wuestemann J, Hupfeld S, Kupitz D, Genseke P, Schenke S, Pech M, et al. Analysis of Bone Scans in Various Tumor Entities Using a Deep-LearningBased Artificial Neural Network Algorithm-Evaluation of Diagnostic Performance. Cancers (Basel) (2020) 12(9):1-13. doi: 10.3390/cancers12092654

132. Diamantis DE, Iakovidis DK, Koulaouzidis A. Look-Behind Fully Convolutional Neural Network for Computer-Aided Endoscopy. BioMed Signal Process Control (2019) 49:192-201. doi: 10.1016/j.bspc.2018.12.005

133. Diamantis DE, Koutsiou D-CC, Iakovidis DK. Staircase Detection Using a Lightweight Look-Behind Fully Convolutional Neural Network. Cham: Springer International Publishing (2019) p. 522-32.

134. Bochkovskiy A, Wang C-Y, Liao H-YM. Yolov4: Optimal Speed and Accuracy of Object Detection. arXiv:200410934 (2020).

135. Redmon J, Divvala S, Girshick R, Farhadi A. You Only Look Once: Unified, Real-Time Object Detection. IEEE (2016) p:779-88. doi: 10.1109/ CVPR.2016.91

136. Borrelli $\mathrm{P}$, Larsson $\mathrm{M}$, Ulén J, Enqvist $\mathrm{O}$, Trägårdh E, Poulsen $\mathrm{MH}$, et al. Artificial Intelligence-Based Detection of Lymph Node Metastases by PET/ CT Predicts Prostate Cancer-Specific Survival. Clin Physiol Funct Imaging (2021) 41(1):62-7. doi: 10.1111/cpf.12666

137. Hartenstein A, Lübbe F, Baur ADJ, Rudolph MM, Furth C, Brenner W, et al. Prostate Cancer Nodal Staging: Using Deep Learning to Predict 68ga-PSMAPositivity From CT Imaging Alone. Sci Rep (2020) 10(1):3398. doi: 10.1038/ s41598-020-60311-z

138. Masoudi S, Mehralivand S, Harmon SA, Lay N, Lindenberg L, Mena E, et al. Deep Learning Based Staging of Bone Lesions From Computed Tomography Scans. IEEE Access (2021) 9:87531-42. doi: 10.1109/ACCESS.2021.3074051

139. Lee JJ, Yang H, Franc BL, Iagaru A, Davidzon GA. Deep Learning Detection of Prostate Cancer Recurrence With 18F-FACBC (Fluciclovine, Axumin ${ }^{\circledR}$ ) Positron Emission Tomography. Eur J Nucl Med Mol Imaging (2020) 47 (13):2992-7. doi: 10.1007/s00259-020-04912-w

140. Steyerberg EW. Clinical Prediction Models: A Practical Approach to Development, Validation, and Updating. New York, NY: Springer-Verlag (2009).

141. Johnson WE, Li C, Rabinovic A. Adjusting Batch Effects in Microarray Expression Data Using Empirical Bayes Methods. Biostatistics (2007) 8 (1):118-27. doi: 10.1093/biostatistics/kxj037

142. Orlhac F, Boughdad S, Philippe C, Stalla-Bourdillon H, Nioche C, Champion L, et al. A Postreconstruction Harmonization Method for Multicenter Radiomic Studies in PET. J Nucl Med (2018) 59(8):1321-8. doi: 10.2967/ jnumed.117.199935

143. Orlhac F, Frouin F, Nioche C, Ayache N, Buvat I. Validation of a Method to Compensate Multicenter Effects Affecting CT Radiomics. Radiology (2019) 291(1):53-9. doi: 10.1148/radiol.2019182023

144. Mahon RN, Ghita M, Hugo GD, Weiss E. ComBat Harmonization for Radiomic Features in Independent Phantom and Lung Cancer Patient Computed Tomography Datasets. Phys Med Biol (2020) 65(1):015010. doi: 10.1088/1361-6560/ab6177

145. Orlhac F, Lecler A, Savatovski J, Goya-Outi J, Nioche C, Charbonneau F, et al. How Can We Combat Multicenter Variability in MR Radiomics? Validation of a Correction Procedure. Eur Radiol (2021) 31(4):2272-80. doi: $10.1007 / \mathrm{s} 00330-020-07284-9$
146. Foy JJ, Robinson KR, Li H, Giger ML, Al-Hallaq H. Armato rSG. Variation in Algorithm Implementation Across Radiomics Software. J Med Imaging (2018) 5(4):44505. doi: 10.1117/1.JMI.5.4.044505

147. Zwanenburg A, Vallières M, Abdalah MA, Aerts HJWL, Anearczyk V, Apte $A$, et al. The Image Biomarker Standardization Initiative: Standardized Quantitative Radiomics for High-Throughput Image-Based Phenotyping. Radiology (2020) 295(2):328-38. doi: 10.1148/radiol.2020191145

148. van Griethuysen JJM, Fedorov A, Parmar C, Hosny A, Aucoin N, Narayan V, et al. Computational Radiomics System to Decode the Radiographic Phenotype. Cancer Res (2017) 77(21):E104-7. doi: 10.1158/0008-5472.CAN-17-0339

149. Pfaehler E, Zwanenburg A, de Jong JR, Boellaard R. RACAT: An Open Source and Easy to Use Radiomics Calculator Tool. PloS One (2019) 14(2): e0212223. doi: 10.1371/journal.pone. 0212223

150. Thomas TS, Pachynski RK. Treatment of Advanced Prostate Cancer. Mo Med (2018) 115(2):156-61.

151. Ronneberger O, Fischer P, Brox T. U-Net: Convolutional Networks for Biomedical Image Segmentation. arXiv:150504597 (2015).

152. Ibtehaz N, Rahman MS. MultiResUNet : Rethinking the U-Net Architecture for Multimodal Biomedical Image Segmentation. Neural Netw (2020) 121:74-87. doi: 10.1016/j.neunet.2019.08.025

153. Weng Y, Zhou T, Li Y, Qiu X. NAS-Unet: Neural Architecture Search for Medical Image Segmentation. IEEE Access (2019) 7:44247-57. doi: 10.1109/ ACCESS.2019.2908991

154. Kostyszyn D, Fechter T, Bartl N, Grosu AL, Gratzke C, Sigle A, et al. Convolutional Neural Network Based Deep-Learning Architecture for Intraprostatic Tumour Contouring on PSMA PET Images in Patients With Primary Prostate Cancer. arXiv:200803201 (2020).

155. Dong X, Lei Y, Tian S, Wang T, Patel P, Curran WJ, et al. Synthetic MRIAided Multi-Organ Segmentation on Male Pelvic CT Using Cycle Consistent Deep Attention Network. Radiother Oncol (2019) 141:192-9. doi: 10.1016/ j.radonc.2019.09.028

156. Kazemifar S, Balagopal A, Nguyen D, McGuire S, Hannan R, Jiang S, et al. Segmentation of the Prostate and Organs at Risk in Male Pelvic CT Images Using Deep Learning. BioMed Phys Eng Express (2018) 4(5):55003. doi: 10.1088/2057-1976/aad100

157. Liu C, Gardner SJ, Wen N, Elshaikh MA, Siddiqui F, Movsas B, et al. Automatic Segmentation of the Prostate on CT Images Using Deep Neural Networks (DNN). Int J Radiat Oncol Biol Phys (2019) 104(4):924-32. doi: 10.1016/j.ijrobp.2019.03.017

158. Clark T, Zhang J, Baig S, Wong A, Haider MA, Khalvati F. Fully Automated Segmentation of Prostate Whole Gland and Transition Zone in DiffusionWeighted MRI Using Convolutional Neural Networks. J Med Imaging (2017) 4(4):41307. doi: 10.1117/1.JMI.4.4.041307

159. Jia H, Xia Y, Song Y, Zhang D, Huang H, Zhang Y, et al. 3d APA-Net: 3d Adversarial Pyramid Anisotropic Convolutional Network for Prostate Segmentation in MR Images. IEEE Trans Med Imaging (2020) 39(2):44757. doi: 10.1109/TMI.2019.2928056

160. Khan Z, Yahya N, Alsaih K, Ali SSA, Meriaudeau F. Evaluation of Deep Neural Networks for Semantic Segmentation of Prostate in T2W MRI. Sensors (2020) 20(11):1-17. doi: 10.3390/s20113183

161. Hinton GE, Srivastava N, Krizhevsky A, Sutskever I, Salakhutdinov RR. Improving Neural Networks by Preventing Co-Adaptation of Feature Detectors. arXiv:12070580 (2012).

162. S Ioffe and C Szegedy eds. Batch Normalization: Accelerating Deep Network Training by Reducing Internal Covariate Shift. In: International Conference on Machine Learning. Lille: PMLR.

163. Castiglioni I, Gallivanone F, Soda P, Avanzo M, Stancanello J, Aiello M, et al. AI-Based Applications in Hybrid Imaging: How to Build Smart and Truly Multi-Parametric Decision Models for Radiomics. Eur J Nucl Med Mol Imaging (2019) 46(13):2673-99. doi: 10.1007/s00259-019-04414-4

Conflict of Interest: The authors declare that the research was conducted in the absence of any commercial or financial relationships that could be construed as a potential conflict of interest.

Publisher's Note: All claims expressed in this article are solely those of the authors and do not necessarily represent those of their affiliated organizations, or those of the publisher, the editors and the reviewers. Any product that may be evaluated in 
this article, or claim that may be made by its manufacturer, is not guaranteed or endorsed by the publisher.

Copyright $\odot 2021$ Kendrick, Francis, Hassan, Rowshanfarzad, Jeraj, Kasisi, Rusanov and Ebert. This is an open-access article distributed under the terms of the Creative
Commons Attribution License (CC BY). The use, distribution or reproduction in other forums is permitted, provided the original author(s) and the copyright owner(s) are credited and that the original publication in this journal is cited, in accordance with accepted academic practice. No use, distribution or reproduction is permitted which does not comply with these terms. 